\title{
Characterizations of Variable Exponent Hardy Spaces via Riesz Transforms
}

\author{
Dachun Yang, Ciqiang Zhuo* and Eiichi Nakai
}

\begin{abstract}
Let $p(\cdot): \mathbb{R}^{n} \rightarrow(0, \infty)$ be a variable exponent function satisfying that

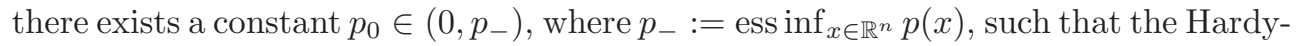
Littlewood maximal operator is bounded on the variable exponent Lebesgue space $L^{p(\cdot) / p_{0}}\left(\mathbb{R}^{n}\right)$. In this article, via investigating relations between boundary valued of harmonic functions on the upper half space and elements of variable exponent Hardy spaces $H^{p(\cdot)}\left(\mathbb{R}^{n}\right)$ introduced by E. Nakai and Y. Sawano and, independently, by D. Cruz-Uribe and L.-A. D. Wang, the authors characterize $H^{p(\cdot)}\left(\mathbb{R}^{n}\right)$ via the first order Riesz transforms when $p_{-} \in\left(\frac{n-1}{n}, \infty\right)$, and via compositions of all the first order Riesz transforms when $p_{-} \in\left(0, \frac{n-1}{n}\right)$.
\end{abstract}

\section{Introduction}

The main purpose of this article is to establish Riesz transform characterizations of the variable exponent Hardy spaces introduced by Nakai and Sawano [14] and, independently, by Cruz-Uribe and Wang [5]. Let $\mathcal{S}\left(\mathbb{R}^{n}\right)$ be the space of all Schwartz functions on $\mathbb{R}^{n}$. Recall that, for all $j \in\{1, \ldots, n\}$, the $j$-th Riesz transform is usually defined by setting, for all $f \in \mathcal{S}\left(\mathbb{R}^{n}\right)$ and $x \in \mathbb{R}^{n}$,

$$
R_{j}(f)(x):=\lim _{\delta \rightarrow 0^{+}} C_{(n)} \int_{\left\{y \in \mathbb{R}^{n}:|y|>\delta\right\}} \frac{y_{j}}{|y|^{n+1}} f(x-y) d y,
$$

here and hereafter, $\delta \rightarrow 0^{+}$means that $\delta \in(0, \infty)$ and $\delta \rightarrow 0, C_{(n)}:=\frac{\Gamma([n+1] / 2)}{\pi^{(n+1) / 2}}$ and $\Gamma$ denotes the Gamma function.

It is well known that the Riesz transform is a natural generalization of the Hilbert transform on the real line $\mathbb{R}$ to the higher dimension Euclidean space $\mathbb{R}^{n}$. Moreover, Riesz transforms are the most typical examples of Calderón-Zygmund operators and have many interesting and useful properties (see, for example, $[9,21,22]$ and their references). Indeed, they are the simplest, non-trivial and "invariant" operators under the acting of the group of

2010 Mathematics Subject Classification. Primary 42B30; Secondary 47B06, 42B35, 42B25.

Key words and phrases. Hardy space, variable exponent, Riesz transform, harmonic function

This project is supported by the National Natural Science Foundation of China (Grant Nos. 11171027 and 11361020), the Specialized Research Fund for the Doctoral Program of Higher Education of China (Grant No. 20120003110003) and the Fundamental Research Funds for Central Universities of China (Grant Nos. 2013YB60 and 2014KJJCA10). The third author is supported by Grant-in-Aid for Scientific Research (B), No. 15H03621, Japan Society for the Promotion of Science.

*Corresponding author 
rotations in the Euclidean space $\mathbb{R}^{n}$, and also constitute typical and important examples of Fourier multipliers. Recall also that, when studying the boundedness of Riesz transforms on Lebesgue spaces $L^{p}\left(\mathbb{R}^{n}\right)$ with $p \in(0,1]$, the Hardy space $H^{p}\left(\mathbb{R}^{n}\right)$ was introduced as a suitable substitute of $L^{p}\left(\mathbb{R}^{n}\right)$ and now plays very important roles in harmonic analysis and partial differential equations (see, for example, $[3,7,13]$ ).

Beside the boundedness of Riesz transforms or, more generally, Calderón-Zygmund type operators on various function spaces including Hardy spaces, the Riesz transform characterizations on Hardy spaces or local Hardy spaces also attract much attention (see, for example, $[2,7,8,18,22,26,27])$. The research on characterizations of Hardy spaces via Riesz transforms originated from Fefferman-Stein's celebrating seminal paper [7] in 1972 and then was extended by Wheeden [27] to the weighted Hardy space $H_{w}^{1}\left(\mathbb{R}^{n}\right)$. Very recently, Cao et al. [2] established Riesz transform characterizations of some Hardy spaces of Musielak-Orlicz type introduced by Ky [11], which essentially extends the results of Wheeden [27] and also the corresponding results of Hardy spaces $H^{p}\left(\mathbb{R}^{n}\right.$ ) (see, for example, [22, p. 123, Proposition 3]). As was well known, when establishing Riesz transform characterizations of Hardy spaces $H^{p}\left(\mathbb{R}^{n}\right)$, one needs to extend the elements of $H^{p}\left(\mathbb{R}^{n}\right)$ to the upper half space $\mathbb{R}_{+}^{n+1}:=\mathbb{R}^{n} \times(0, \infty)$ via Poisson integrals. This extension in turn has a close relationship with the analytical definition of $H^{p}\left(\mathbb{R}^{n}\right)$ which is the key starting point for the study on the Hardy space, before one paid attention to the real-variable theory of $H^{p}\left(\mathbb{R}^{n}\right)$ (see $[12,20,23,24]$ ).

It is known that the Hardy space $H^{p}\left(\mathbb{R}^{n}\right)$ can be characterized by the first order Riesz transforms when $p \in\left(\frac{n-1}{n}, \infty\right)$ (see [7] or [22, p. 123, Proposition 3]) and by the higher order Riesz transforms when $p \in\left(0, \frac{n-1}{n}\right]$ (see [7, p. 168]). Precisely, denote by $\mathcal{S}^{\prime}\left(\mathbb{R}^{n}\right)$ the dual space of $\mathcal{S}\left(\mathbb{R}^{n}\right)$, namely, the space of all tempered distributions. Recall that a distribution $f \in \mathcal{S}^{\prime}\left(\mathbb{R}^{n}\right)$ is called a distribution restricted at infinity if there exists a positive number $r$ large enough such that, for all $\phi \in \mathcal{S}\left(\mathbb{R}^{n}\right), f * \phi \in L^{r}\left(\mathbb{R}^{n}\right)$. Then the following conclusions hold true, which were obtained in [7] (see also [22, p. 123, Proposition 3 and p. 133, Item 5.16]).

Theorem 1.1 ([7]). Let $\phi \in \mathcal{S}\left(\mathbb{R}^{n}\right)$ satisfy $\int_{\mathbb{R}^{n}} \phi(x) d x=1$.

(i) If $p \in\left(\frac{n-1}{n}, \infty\right)$, then $f \in H^{p}\left(\mathbb{R}^{n}\right)$ if and only if $f$ is a distribution restricted at infinity and there exists a positive constant $A_{1}$ such that, for all $\epsilon \in(0, \infty)$,

$$
\left\|f * \phi_{\epsilon}\right\|_{L^{p}\left(\mathbb{R}^{n}\right)}+\sum_{j=1}^{n}\left\|R_{j}(f) * \phi_{\epsilon}\right\|_{L^{p}\left(\mathbb{R}^{n}\right)} \leq A_{1}
$$

where, for all $x \in \mathbb{R}^{n}, \phi_{\epsilon}(x):=\frac{1}{\epsilon^{n}} \phi\left(\frac{x}{\epsilon}\right)$; moreover, $\|f\|_{H^{p}\left(\mathbb{R}^{n}\right)} \sim A_{1}$ with implicit equivalent positive constants independent of $f$ and $\epsilon$.

(ii) If $m \in \mathbb{N}$ and $p \in\left(\frac{n-1}{n+m-1}, \infty\right)$, then $f \in H^{p}\left(\mathbb{R}^{n}\right)$ if and only if $f$ is a distribution restricted at infinity and there exists a positive constant $A_{2}$ such that, for all $\epsilon \in(0, \infty)$,

$$
\left\|f * \phi_{\epsilon}\right\|_{L^{p}\left(\mathbb{R}^{n}\right)}+\sum_{k=1}^{m} \sum_{j_{1}, \ldots, j_{k}=1}^{n}\left\|R_{j_{1}} \cdots R_{j_{k}}(f) * \phi_{\epsilon}\right\|_{L^{p}\left(\mathbb{R}^{n}\right)} \leq A_{2} ;
$$

moreover, $\|f\|_{H^{p}\left(\mathbb{R}^{n}\right)} \sim A_{2}$ with implicit equivalent positive constants independent of $f$ and $\epsilon$. 
In this article, we are devoted to extending conclusions of Theorem 1.1 to the variable exponent Hardy space $H^{p(\cdot)}\left(\mathbb{R}^{n}\right)$ which was first studied by Nakai and Sawano [14] and, independently, by Cruz-Uribe and Wang [5]. Recall that the variable exponent Lebesgue space $L^{p(\cdot)}\left(\mathbb{R}^{n}\right)$, with a variable exponent function $p(\cdot): \mathbb{R}^{n} \rightarrow(0, \infty)$, consists of all measurable functions $f$ such that $\int_{\mathbb{R}^{n}}|f(x)|^{p(x)} d x<\infty$. As a generalization of classical Lebesgue spaces, variable exponent Lebesgue spaces were introduced by Orlicz [17] in 1931, however, they have been the subject of more intensive study since the early 1990s, because of their intrinsic interest for applications into harmonic analysis, partial differential equations and variational integrals with nonstandard growth conditions (see $[4,6,10]$ and their references). The variable exponent Hardy space $H^{p(\cdot)}\left(\mathbb{R}^{n}\right)$ extends not only the variable exponent Lebesgue space $L^{p(\cdot)}\left(\mathbb{R}^{n}\right)$ but also the Hardy space $H^{p}\left(\mathbb{R}^{n}\right)$ with constant exponent.

Particularly, Nakai and Sawano [14] introduced Hardy spaces with variable exponents, $H^{p(\cdot)}\left(\mathbb{R}^{n}\right)$, and established their atomic characterizations which were further applied to consider their duals. Later, in [19], Sawano extended the atomic characterization of the space $H^{p(\cdot)}\left(\mathbb{R}^{n}\right)$ in [14], which also improves the corresponding result in [14], and gave out more applications including the boundedness of several operators. Moreover, Zhuo et al. [31] established their equivalent characterizations via intrinsic square functions, including the intrinsic Lusin area function, the intrinsic $g$-function and the intrinsic $g_{\lambda}^{*}$-function.

Independently, Cruz-Uribe and Wang [5] also investigated the variable exponent Hardy space $H^{p(\cdot)}\left(\mathbb{R}^{n}\right)$ with $p(\cdot)$ satisfying some slightly weaker conditions than those used in [14]. Moreover, in [5], characterizations of $H^{p(\cdot)}\left(\mathbb{R}^{n}\right)$ in terms of radial or non-tangential maximal functions or atoms were established, and the boundedness of singular integral operators were also obtained.

A measurable function $p(\cdot): \mathbb{R}^{n} \rightarrow(0, \infty)$ is called a variable exponent. For any variable exponent $p(\cdot)$, let

$$
p_{-}:=\operatorname{essinf}_{x \in \mathbb{R}^{n}} p(x) \quad \text { and } \quad p_{+}:=\operatorname{ess}_{x \in \mathbb{R}^{n}} \sup p(x) .
$$

Denote by $\mathcal{P}\left(\mathbb{R}^{n}\right)$ the collection of variable exponents $p(\cdot)$ satisfying $0<p_{-} \leq p_{+}<\infty$.

For a measurable function $f$ on $\mathbb{R}^{n}$ and a variable exponent $p(\cdot) \in \mathcal{P}\left(\mathbb{R}^{n}\right)$, the modular $\varrho_{p(\cdot)}(f)$ of $f$ is defined by setting $\varrho_{p(\cdot)}(f):=\int_{\mathbb{R}^{n}}|f(x)|^{p(x)} d x$ and the Luxemburg (also called Luxemburg-Nakano) quasi-norm is given by

$$
\|f\|_{L^{p(\cdot)}\left(\mathbb{R}^{n}\right)}:=\inf \left\{\lambda \in(0, \infty): \varrho_{p(\cdot)}(f / \lambda) \leq 1\right\} .
$$

Then the variable exponent Lebesgue space $L^{p(\cdot)}\left(\mathbb{R}^{n}\right)$ is defined to be the set of all measurable functions $f$ such that $\varrho_{p(\cdot)}(f)<\infty$ equipped with the quasi-norm $\|f\|_{L^{p(\cdot)}\left(\mathbb{R}^{n}\right)}$.

Remark 1.2. Let $p(\cdot) \in \mathcal{P}\left(\mathbb{R}^{n}\right)$.

(i) If $p_{-} \in[1, \infty)$, then $L^{p(\cdot)}\left(\mathbb{R}^{n}\right)$ is a Banach space (see [6, Theorem 3.2.7]). Particularly, for all $\lambda \in \mathbb{C}$ and $f \in L^{p(\cdot)}\left(\mathbb{R}^{n}\right),\|\lambda f\|_{L^{p(\cdot)}\left(\mathbb{R}^{n}\right)}=|\lambda|\|f\|_{L^{p(\cdot)}\left(\mathbb{R}^{n}\right)}$ and, for all $f, g \in L^{p(\cdot)}\left(\mathbb{R}^{n}\right)$,

$$
\|f+g\|_{L^{p(\cdot)}\left(\mathbb{R}^{n}\right)} \leq\|f\|_{L^{p(\cdot)\left(\mathbb{R}^{n}\right)}}+\|g\|_{L^{p(\cdot)}\left(\mathbb{R}^{n}\right)} .
$$


(ii) For any non-trivial function $f \in L^{p(\cdot)}\left(\mathbb{R}^{n}\right)$, it holds true that $\varrho_{p(\cdot)}\left(f /\|f\|_{L^{p(\cdot)}\left(\mathbb{R}^{n}\right)}\right)=1$ (see, for example, [4, Proposition 2.21]).

(iii) If $\int_{\mathbb{R}^{n}}[|f(x)| / \delta]^{p(x)} d x \leq c$ for some $\delta \in(0, \infty)$ and some positive constant $c$ independent of $\delta$, then it is easy to see that $\|f\|_{L^{p(\cdot)}\left(\mathbb{R}^{n}\right)} \leq C \delta$, where $C$ is a positive constant independent of $\delta$, but depending on $p_{-}$(or $p_{+}$) and $c$.

(iv) (The Hölder inequality) Assume that $p_{-} \in[1, \infty)$. It was proved in $[6$, Lemma 3.2.20] (see also [4, Theorem 2.26]) that, if $f \in L^{p(\cdot)}\left(\mathbb{R}^{n}\right)$ and $g \in L^{p^{*}(\cdot)}\left(\mathbb{R}^{n}\right)$, then $f g \in$ $L^{1}\left(\mathbb{R}^{n}\right)$ and

$$
\int_{\mathbb{R}^{n}}|f(x) g(x)| d x \leq C\|f\|_{L^{p(\cdot)}\left(\mathbb{R}^{n}\right)}\|g\|_{L^{p^{*}(\cdot)}\left(\mathbb{R}^{n}\right)},
$$

where $p^{*}(\cdot)$ denotes the dual variable exponent of $p(\cdot)$ defined by $\frac{1}{p(x)}+\frac{1}{p^{*}(x)}=1$ for all $x \in \mathbb{R}^{n}$, with $1 / \infty=0$, and $C$ is a positive constant depending on $p_{-}$or $p_{+}$, but independent of $f$ and $g$.

(v) Assume that $p_{-} \in[1, \infty)$. For any given measurable function $f$ on $\mathbb{R}^{n}$, let

$$
\|f\|_{L^{p(\cdot)}\left(\mathbb{R}^{n}\right)}^{*}:=\sup \left|\int_{\mathbb{R}^{n}} f(x) g(x) d x\right|,
$$

where the supremum is taken over all $g \in L^{p^{*}(\cdot)}\left(\mathbb{R}^{n}\right)$ satisfying $\|g\|_{L^{p^{*}(\cdot)}\left(\mathbb{R}^{n}\right)} \leq 1$. Then it was proved in $\left[4\right.$, Theorem 2.34] that, for all $f \in L^{p(\cdot)}\left(\mathbb{R}^{n}\right),\|f\|_{L^{p(\cdot)}\left(\mathbb{R}^{n}\right)} \sim\|f\|_{L^{p(\cdot)}\left(\mathbb{R}^{n}\right)}$ with the implicit equivalent positive constants independent of $f$.

For all $r \in(0, \infty)$, denote by $L_{\text {loc }}^{r}\left(\mathbb{R}^{n}\right)$ the set of all locally $r$-integrable functions on $\mathbb{R}^{n}$ and, for any measurable set $E \subset \mathbb{R}^{n}$, by $L^{r}(E)$ the set of all measurable functions $f$ such that

$$
\|f\|_{L^{r}(E)}:=\left\{\int_{E}|f(x)|^{r} d x\right\}^{1 / r}<\infty .
$$

Recall that the Hardy-Littlewood maximal operator $\mathcal{M}$ is defined by setting, for all $f \in$ $L_{\text {loc }}^{1}\left(\mathbb{R}^{n}\right)$ and $x \in \mathbb{R}^{n}$,

$$
\mathcal{M}(f)(x):=\sup _{B \ni x} \frac{1}{|B|} \int_{B}|f(y)| d y
$$

where the supremum is taken over all balls $B$ of $\mathbb{R}^{n}$ containing $x$.

For any $p(\cdot) \in \mathcal{P}\left(\mathbb{R}^{n}\right), p(\cdot)$ is said to belong to $\mathcal{M P}\left(\mathbb{R}^{n}\right)$ if there exists $p_{0} \in\left(0, p_{-}\right)$such that the maximal operator $\mathcal{M}$ is bounded on $L^{p(\cdot) / p_{0}}\left(\mathbb{R}^{n}\right)$.

For any $N \in \mathbb{N}$, let

$$
\mathcal{F}_{N}\left(\mathbb{R}^{n}\right):=\left\{\psi \in \mathcal{S}\left(\mathbb{R}^{n}\right): \sup _{\alpha, \beta \in \mathbb{Z}_{+}^{n},|\alpha|,|\beta| \leq N} \sup _{x \in \mathbb{R}^{n}}\left|x^{\alpha} \partial^{\beta} \psi(x)\right| \leq 1\right\},
$$

where, for all $\beta:=\left(\beta_{1}, \ldots, \beta_{n}\right) \in \mathbb{Z}_{+}^{n},|\beta|:=\beta_{1}+\cdots+\beta_{n}$ and $\partial^{\beta}:=\left(\frac{\partial}{\partial x_{1}}\right)^{\beta_{1}} \cdots\left(\frac{\partial}{\partial x_{n}}\right)^{\beta_{n}}$. In what follows, for all $\psi \in \mathcal{S}\left(\mathbb{R}^{n}\right), t \in(0, \infty)$ and $\xi \in \mathbb{R}^{n}$, let $\psi_{t}(\xi):=t^{-n} \psi(\xi / t)$. Then, for all $f \in \mathcal{S}^{\prime}\left(\mathbb{R}^{n}\right)$, the radial grand maximal function $f_{N,+}^{*}$ is defined by setting, for all $x \in \mathbb{R}^{n}$,

$$
f_{N,+}^{*}(x):=\sup _{\psi \in \mathcal{F}_{N}\left(\mathbb{R}^{n}\right)} M_{\psi,+}^{*}(f)(x),
$$


where, $M_{\psi,+}^{*}(f)$ denotes the radial maximal function of $f$ defined by setting, for all $x \in \mathbb{R}^{n}$,

$$
M_{\psi,+}^{*}(f)(x):=\sup _{t \in(0, \infty)}\left|f * \psi_{t}(x)\right| .
$$

Definition 1.3. Let $p(\cdot) \in \mathcal{M P}\left(\mathbb{R}^{n}\right)$ with $p_{0} \in\left(0, p_{-}\right)$and $N \in\left(\frac{n}{p_{0}}+n+1, \infty\right)$. Then the variable exponent Hardy space $H^{p(\cdot)}\left(\mathbb{R}^{n}\right)$ is defined to be the set of all $f \in \mathcal{S}^{\prime}\left(\mathbb{R}^{n}\right)$ such that $f_{N,+}^{*} \in L^{p(\cdot)}\left(\mathbb{R}^{n}\right)$ endowed with the quasi-norm $\|f\|_{H^{p(\cdot)}\left(\mathbb{R}^{n}\right)}:=\left\|f_{N,+}^{*}\right\|_{L^{p(\cdot)}\left(\mathbb{R}^{n}\right)}$.

Remark 1.4. (i) We point out that the variable exponent Hardy space was first studied by Nakai and Sawano [14]. However, in [14], the variable exponent $p(\cdot) \in \mathcal{P}\left(\mathbb{R}^{n}\right)$ is required to satisfy globally Hölder continuous condition, namely, there exist positive constants $C_{\log }(p)$ and $C_{\infty}$, and $p_{\infty} \in \mathbb{R}$ such that, for all $x, y \in \mathbb{R}^{n}$,

$$
|p(x)-p(y)| \leq \frac{C_{\log }(p)}{\log (e+1 /|x-y|)}
$$

and

$$
\left|p(x)-p_{\infty}\right| \leq \frac{C_{\infty}}{\log (e+|x|)}
$$

(ii) Independently, Cruz-Uribe and Wang [5] also studied the variable exponent Hardy space, but they only assume that $p(\cdot) \in \mathcal{M P}\left(\mathbb{R}^{n}\right)$. We point out that, if $p(\cdot)$ satisfies the globally Hölder continuous condition, then $p(\cdot) \in \mathcal{M P}\left(\mathbb{R}^{n}\right)$; see $[4,6]$. Therefore, the assumption on $p(\cdot)$ in [5] is slightly weaker than that in [14]. In the present article, we only assume that $p(\cdot) \in \mathcal{M P}\left(\mathbb{R}^{n}\right)$.

(iii) Let $p(\cdot) \in \mathcal{P}\left(\mathbb{R}^{n}\right)$. If the maximal operator $\mathcal{M}$ is bounded on $L^{p(\cdot)}\left(\mathbb{R}^{n}\right)$, then, for all $s \in(1, \infty), \mathcal{M}$ is also bounded on $L^{s p(\cdot)}\left(\mathbb{R}^{n}\right)$ (see [5, Lemma 2.12]).

The main results of this article are stated as follows.

Theorem 1.5. Let $p(\cdot) \in \mathcal{M P}\left(\mathbb{R}^{n}\right)$ with $p_{-} \in\left(\frac{n-1}{n}, \infty\right), f \in \mathcal{S}^{\prime}\left(\mathbb{R}^{n}\right)$ and $\phi \in \mathcal{S}\left(\mathbb{R}^{n}\right)$ satisfy $\int_{\mathbb{R}^{n}} \phi(x) d x=1$. Then the following items are equivalent:

(i) $f \in H^{p(\cdot)}\left(\mathbb{R}^{n}\right)$;

(ii) $f$ is a distribution restricted at infinity and there exists a positive constant $A_{3}$ such that, for all $\epsilon \in(0, \infty)$,

$$
\left\|f * \phi_{\epsilon}\right\|_{L^{p(\cdot)\left(\mathbb{R}^{n}\right)}}+\sum_{j=1}^{n}\left\|R_{j}(f) * \phi_{\epsilon}\right\|_{L^{p(\cdot)}\left(\mathbb{R}^{n}\right)} \leq A_{3} .
$$

Moreover, there exists a positive constant $C$, independent of $f$, such that

$$
C^{-1}\|f\|_{H^{p(\cdot)}\left(\mathbb{R}^{n}\right)} \leq A_{3} \leq C\|f\|_{H^{p(\cdot)\left(\mathbb{R}^{n}\right)}} .
$$

Furthermore, if $p_{-} \in[1, \infty)$, then (1.2) can be replaced by

$$
\|f\|_{L^{p(\cdot)}\left(\mathbb{R}^{n}\right)}+\sum_{j=1}^{n}\left\|R_{j}(f)\right\|_{L^{p(\cdot)}\left(\mathbb{R}^{n}\right)} \leq A_{3} .
$$


Theorem 1.6. Let $m \in \mathbb{N} \cap[2, \infty)$ and $p(\cdot) \in \mathcal{M P}\left(\mathbb{R}^{n}\right)$ satisfy $p_{-} \in\left(\frac{n-1}{n+m-1}, \infty\right)$, and let $f \in \mathcal{S}^{\prime}\left(\mathbb{R}^{n}\right)$ and $\phi \in \mathcal{S}\left(\mathbb{R}^{n}\right)$ satisfy $\int_{\mathbb{R}^{n}} \phi(x) d x=1$. Then the following items are equivalent:

(i) $f \in H^{p(\cdot)}\left(\mathbb{R}^{n}\right)$;

(ii) $f$ is a distribution restricted at infinity and there exists a positive constant $A_{4}$ such that, for all $\varepsilon \in(0, \infty)$,

$$
\left\|f * \phi_{\varepsilon}\right\|_{L^{p(\cdot)}\left(\mathbb{R}^{n}\right)}+\sum_{k=1}^{m} \sum_{j_{1}, \ldots, j_{k}=1}^{n}\left\|R_{j_{1}} \cdots R_{j_{k}}(f) * \phi_{\varepsilon}\right\|_{L^{p(\cdot)}\left(\mathbb{R}^{n}\right)} \leq A_{4} .
$$

Moreover, there exists a positive constant $C$, independent of $f$, such that

$$
C^{-1}\|f\|_{H^{p(\cdot)}\left(\mathbb{R}^{n}\right)} \leq A_{4} \leq C\|f\|_{H^{p(\cdot)\left(\mathbb{R}^{n}\right)}} .
$$

Remark 1.7. (i) Obviously, when $p(\cdot) \equiv p$ with $p \in\left(\frac{n-1}{n}, \infty\right)$, Theorem 1.5 is just Theorem 1.1(i) (see also $\left[22\right.$, p. 123, Proposition 3]) and, when $p(\cdot) \equiv p$ with $p \in\left(\frac{n-1}{n+m+1}, \infty\right)$, Theorem 1.6 is just Theorem 1.1(ii) (see also [22, p. 122, Item 5.16]).

(ii) Observer that, for any $p(\cdot) \in \mathcal{P}\left(\mathbb{R}^{n}\right)$, there exists an $m \in \mathbb{N}$ such that $p_{-} \in$ $\left(\frac{n-1}{n+m-1}, \infty\right)$. Thus, in Theorem 1.6, we actually obtain the Riesz transform characterization of $H^{p(\cdot)}\left(\mathbb{R}^{n}\right)$ for all $p(\cdot) \in \mathcal{M P}\left(\mathbb{R}^{n}\right)$ with $p_{-} \in(0, \infty)$ via different choices of $m \in \mathbb{N}$.

(iii) If $f$ is a distribution restricted at infinity, then, for any $j \in\{1, \ldots, n\}, R_{j}(f)$ is well defined as a distribution (see [22, p. 123]).

(iv) Recall that, in [2], Cao et al. characterized the Musielak-Orlicz Hardy space $H^{\varphi}\left(\mathbb{R}^{n}\right)$, which was introduced by Ky [11], via Riesz transforms. Observe that, if

$$
\varphi(x, t):=t^{p(x)} \quad \text { for all } \quad x \in \mathbb{R}^{n} \text { and } t \in(0, \infty)
$$

then $H^{\varphi}\left(\mathbb{R}^{n}\right)=H^{p(\cdot)}\left(\mathbb{R}^{n}\right)$. However, a general Musielak-Orlicz function $\varphi$ satisfying all the assumptions in [11] (and hence [2]) may not have the form as in (1.4). On the other hand, it was proved in $[29$, Remark 2.23(iii)] that there exists a variable exponent $p(\cdot)$ satisfying the globally Hölder continuous condition and hence belonging to $\mathcal{M P}\left(\mathbb{R}^{n}\right)$, but $t^{p(\cdot)}$ is not a uniformly Muckenhoupt weight, which was required in [11] (and hence [2]). Therefore, the Musielak-Orlicz Hardy space $H^{\varphi}\left(\mathbb{R}^{n}\right)$ in [11] (and hence [2]) and the variable exponent Hardy space $H^{p(\cdot)}\left(\mathbb{R}^{n}\right)$ in [5] (and hence in the present article) can not cover each other.

This article is organized as follows.

In Section 2, we aim at giving out the proofs of Theorems 1.5 and 1.6. To this end, via borrowing some ideas used in the proof of Theorem 1.1, we not only make full use of the non-tangential and the radial maximal function characterizations of $H^{p(\cdot)}\left(\mathbb{R}^{n}\right)$, but also draw supports from properties of harmonic functions.

Precisely, in Subsection 2.1, we first recall some known results on $H^{p(\cdot)}\left(\mathbb{R}^{n}\right)$ including its characterizations in terms of the non-tangential maximal function corresponding to Poisson kernels in Lemma 2.2 below (see also [5, Theorem 3.1]) and the non-tangential grand maximal function in Lemma 2.4 below (see also [31, Proposition 2.1]). Using these characterizations, we investigate relations between harmonic functions and Poisson integrals 
of elements of $H^{p(\cdot)}\left(\mathbb{R}^{n}\right)$ in Proposition 2.5 below, which are further applied in Lemma 2.7 below to find the harmonic majorant of harmonic vectors satisfying the generalized Cauchy-Riemann equation (2.6) below. Moreover, the boundary value of such a harmonic vector in turn determines a harmonic function $u$ such that its non-tangential maximal function belongs to $L^{p(\cdot)}\left(\mathbb{R}^{n}\right)$ (see Proposition 2.6 below). To prove Proposition 2.6, we need to show that the set $H^{p(\cdot)}\left(\mathbb{R}^{n}\right) \cap L^{1+r}\left(\mathbb{R}^{n}\right)$ with $r \in\left[p_{+}, \infty\right)$ is dense in $H^{p(\cdot)}\left(\mathbb{R}^{n}\right)$ via the Poisson kernels, which improves [5, Proposition 4.2] in which Cruz-Uribe and Wang proved that $H^{p(\cdot)}\left(\mathbb{R}^{n}\right) \cap L_{\text {loc }}^{1}\left(\mathbb{R}^{n}\right)$ is dense in $H^{p(\cdot)}\left(\mathbb{R}^{n}\right)$ and may be of independent interest. The boundedness of Riesz transforms on $H^{p(\cdot)}\left(\mathbb{R}^{n}\right)$, which is essentially established by Cruz-Uribe and Wang in [5], also plays a key role in the proof of Theorem 1.5.

In Subsection 2.2, by some arguments similar to those used in the proof of Theorem 1.5, we prove Theorem 1.6 via tensor-valued functions. We point out that one of important facts used in the proofs of Theorems 1.5 and 1.6 is that, for all $f \in L^{p(\cdot)}\left(\mathbb{R}^{n}\right)$,

$$
\int_{\mathbb{R}^{n}}\left[|f(x)| /\|f\|_{L^{p(\cdot)}\left(\mathbb{R}^{n}\right)}\right]^{p(x)} d x=1,
$$

if $0<p_{-} \leq p_{+}<\infty$, which is well known (see [4, Proposition 2.21]). Another important fact used in the proofs of Theorems 1.5 and 1.6 is that, for any $f \in H^{p(\cdot)}\left(\mathbb{R}^{n}\right)$ and $t \in(0, \infty)$, the Poisson integral $f * P_{t} \in L^{r}\left(\mathbb{R}^{n}\right)$ as long as $r \in\left[p_{+}, \infty\right)$, which is proved in Lemma 2.9(ii) below.

Different from the strategy used in the case of Musielak-Orlicz Hardy spaces in [2], we do not need to restrict the working space to be $H^{p(\cdot)}\left(\mathbb{R}^{n}\right) \cap L^{2}\left(\mathbb{R}^{n}\right)$ to guarantee that $\left\{R_{j}(f)\right\}_{j=1}^{n}$ make sense. Indeed, for any $f \in H^{p(\cdot)}\left(\mathbb{R}^{n}\right)$, we can prove that $f$ is a distribution restricted at infinity (see Lemma 2.9(i)), which is not clear whether it is true for elements of Musielak-Orlicz Hardy spaces or not. Thus, for any $j \in\{1, \ldots, n\}$ and $f \in H^{p(\cdot)}\left(\mathbb{R}^{n}\right), R_{j}(f)$ is well defined as a distribution according to Remark 1.7 (iii).

Finally, we make some conventions on notation. Let $\mathbb{N}:=\{1,2, \ldots\}$ and $\mathbb{Z}_{+}:=\mathbb{N} \cup\{0\}$. We denote by $C$ a positive constant which is independent of the main parameters, but may vary from line to line. The symbol $A \lesssim B$ means $A \leq C B$. If $A \lesssim B$ and $B \lesssim A$, then we write $A \sim B$. If $E$ is a subset of $\mathbb{R}^{n}$, we denote by $\chi_{E}$ its characteristic function and by $E^{\complement}$ the set $\mathbb{R}^{n} \backslash E$. For all $r \in(0, \infty)$ and $x \in \mathbb{R}^{n}$, let $B(x, r):=\left\{y \in \mathbb{R}^{n}:|x-y|<r\right\}$.

\section{Proofs of main results}

This section is devoted to the proofs of of Theorems 1.5 and 1.6. Precisely, Theorem 1.5 is proved in Subsection 2.1 and the proof of Theorem 1.6 is presented out in Subsection 2.2 .

\subsection{The case $p_{-} \in\left(\frac{n-1}{n}, \infty\right)$}

In this subsection, we prove Theorem 1.5. To this end, we need more preparations and begin with the following notation.

A distribution $f \in \mathcal{S}^{\prime}\left(\mathbb{R}^{n}\right)$ is called a bounded distribution if, for all $\psi \in \mathcal{S}\left(\mathbb{R}^{n}\right), f * \psi \in$ $L^{\infty}\left(\mathbb{R}^{n}\right)$. For a bounded distribution $f$, its non-tangential maximal function is defined by 
setting, for all $x \in \mathbb{R}^{n}$,

$$
f_{P}^{*}(x):=\sup _{(y, t) \in \mathbb{R}_{+}^{n+1},|x-y|<t}\left|f * P_{t}(y)\right|,
$$

where $P_{t}$ denotes the Poisson kernel, which is defined by setting, for all $(x, t) \in \mathbb{R}_{+}^{n+1}$,

$$
P_{t}(x):=\frac{\Gamma([n+1] / 2)}{\pi^{(n+1) / 2}} \frac{t}{\left(t^{2}+|x|^{2}\right)^{(n+1) / 2}} .
$$

Remark 2.1. It is known that, if $f$ is a bounded distribution, then $f * P_{t}$ is well defined. Indeed, by [22, p. 90], we see that there exist $\psi^{(1)}, \psi^{(2)} \in \mathcal{S}\left(\mathbb{R}^{n}\right)$ and $h \in L^{1}\left(\mathbb{R}^{n}\right)$ such that, for all $t \in(0, \infty)$,

$$
P_{t}=\left(\psi^{(1)}\right)_{t} * h_{t}+\left(\psi^{(2)}\right)_{t}
$$

Thus, if $f$ is a bounded distribution, then $f * P_{t}=f *\left(\psi^{(1)}\right)_{t} * h_{t}+f *\left(\psi^{(2)}\right)_{t}$ and hence $f * P_{t}$ is well defined. Moreover, the function $u(x, t):=f * P_{t}(x)$ for $(x, t) \in \mathbb{R}_{+}^{n+1}$ is harmonic on $\mathbb{R}_{+}^{n+1}$ (see $[22$, p. 90]).

The following conclusion was obtained in [5, Theorem 3.1].

Lemma 2.2. Let $p(\cdot) \in \mathcal{M P}\left(\mathbb{R}^{n}\right)$ and $f \in \mathcal{S}^{\prime}\left(\mathbb{R}^{n}\right)$. Then the following three items are equivalent:

(i) $f \in H^{p(\cdot)}\left(\mathbb{R}^{n}\right)$;

(ii) there exists $\Phi \in \mathcal{S}\left(\mathbb{R}^{n}\right)$ with $\int_{\mathbb{R}^{n}} \Phi(x) d x \neq 0$ such that $M_{\Phi,+}^{*}(f) \in L^{p(\cdot)}\left(\mathbb{R}^{n}\right)$;

(iii) $f$ is a bounded distribution and $f_{P}^{*} \in L^{p(\cdot)}\left(\mathbb{R}^{n}\right)$.

Moreover,

$$
\|f\|_{H^{p(\cdot)}\left(\mathbb{R}^{n}\right)} \sim\left\|M_{\Phi,+}^{*}(f)\right\|_{L^{p(\cdot)}\left(\mathbb{R}^{n}\right)} \sim\left\|f_{P}^{*}\right\|_{L^{p(\cdot)}\left(\mathbb{R}^{n}\right)},
$$

where the implicit equivalent positive constants are independent of $f$.

Remark 2.3. If $f \in H^{p(\cdot)}\left(\mathbb{R}^{n}\right)$, then, by Lemma 2.2(iii), $f$ is a bounded distribution. Thus, for any $f \in H^{p(\cdot)}\left(\mathbb{R}^{n}\right), f * P_{t}$ is well defined by Remark 2.1.

For all $N \in \mathbb{N}$ and $f \in \mathcal{S}^{\prime}\left(\mathbb{R}^{n}\right)$, the non-tangential grand maximal function, $f_{N, \nabla}^{*}$, of $f$ is defined by setting, for all $x \in \mathbb{R}^{n}, f_{N, \nabla}^{*}(x):=\sup _{\psi \in \mathcal{S}_{N}\left(\mathbb{R}^{n}\right)} M_{\psi, \nabla}^{*}(f)(x)$, where $M_{\psi, \nabla}^{*}(f)$ denotes the non-tangential maximal function of $f$ defined by setting, for all $y \in \mathbb{R}^{n}$,

$$
M_{\psi, \nabla}^{*}(f)(y):=\sup _{t \in(0, \infty),|\xi-y|<t}\left|f * \psi_{t}(\xi)\right| .
$$

We have the following equivalent characterization of $H^{p(\cdot)}\left(\mathbb{R}^{n}\right)$ via non-tangential grand maximal functions, whose proof can be found in [31, Proposition 2.1] (see also [5, Lemma $7.9])$. 
Lemma 2.4. Let $p(\cdot) \in \mathcal{M P}\left(\mathbb{R}^{n}\right)$ with $p_{0} \in\left(0, p_{-}\right)$and $N \in\left(\frac{n}{p_{0}}+n+1, \infty\right)$. Then $f \in H^{p(\cdot)}\left(\mathbb{R}^{n}\right)$ if and only if $f \in \mathcal{S}^{\prime}\left(\mathbb{R}^{n}\right)$ and $f_{N, \nabla}^{*} \in L^{p(\cdot)}\left(\mathbb{R}^{n}\right)$; moreover, there exists a positive constant $C$ such that, for all $f \in H^{p(\cdot)}\left(\mathbb{R}^{n}\right)$,

$$
C^{-1}\left\|f_{N, \nabla}^{*}\right\|_{L^{p(\cdot)}\left(\mathbb{R}^{n}\right)} \leq\|f\|_{H^{p(\cdot)}\left(\mathbb{R}^{n}\right)} \leq C\left\|f_{N, \nabla}^{*}\right\|_{L^{p(\cdot)}\left(\mathbb{R}^{n}\right)} .
$$

Let $u$ be a measurable function on $\mathbb{R}_{+}^{n+1}$. Then its non-tangential maximal function $u^{*}$ is defined by setting, for all $x \in \mathbb{R}^{n}$,

$$
u^{*}(x):=\sup _{t \in(0, \infty),|y-x|<t}|u(y, t)| .
$$

Recall that a function $u$ on $\mathbb{R}_{+}^{n+1}$ is said to be harmonic if, for all $t \in(0, \infty)$ and $x:=\left(x_{1}, \ldots, x_{n}\right) \in \mathbb{R}^{n}, \partial_{t}^{2} u(x, t)+\sum_{j=1}^{n} \partial_{x_{j}}^{2} u(x, t)=0$.

On relations between harmonic functions and Poisson integrals of elements of the variable exponent Hardy space $H^{p(\cdot)}\left(\mathbb{R}^{n}\right)$, we have the following conclusion.

Proposition 2.5. Let $p(\cdot) \in \mathcal{M P}\left(\mathbb{R}^{n}\right)$ and $u$ be a harmonic function on $\mathbb{R}_{+}^{n+1}$. Then $u^{*} \in L^{p(\cdot)}\left(\mathbb{R}^{n}\right)$ if and only if there exists $f \in H^{p(\cdot)}\left(\mathbb{R}^{n}\right)$ such that, for all $(x, t) \in \mathbb{R}_{+}^{n+1}$, $u(x, t)=f * P_{t}(x)$. Moreover, there exists a positive constant $C$, independent of $f$ and $u$, such that

$$
C^{-1}\|f\|_{H^{p(\cdot)}\left(\mathbb{R}^{n}\right)} \leq\left\|u^{*}\right\|_{L^{p(\cdot)}\left(\mathbb{R}^{n}\right)} \leq C\|f\|_{H^{p(\cdot)\left(\mathbb{R}^{n}\right)}} .
$$

Proof. Assume that there exists $f \in H^{p(\cdot)}\left(\mathbb{R}^{n}\right)$ such that $u(x, t)=f * P_{t}(x)$ for all $(x, t) \in$ $\mathbb{R}_{+}^{n+1}$. Then, by Lemma 2.2 , we see that $f_{P}^{*} \in L^{p(\cdot)}\left(\mathbb{R}^{n}\right)$, which, together with the fact that $u^{*}=f_{P}^{*}$, implies that $u^{*} \in L^{p(\cdot)}\left(\mathbb{R}^{n}\right)$ and $\left\|u^{*}\right\|_{L^{p(\cdot)}\left(\mathbb{R}^{n}\right)} \lesssim\|f\|_{H^{p(\cdot)}\left(\mathbb{R}^{n}\right)}$.

Conversely, suppose that $u^{*} \in L^{p(\cdot)}\left(\mathbb{R}^{n}\right)$. We first claim that $u$ is bounded in any half space $\left\{(x, t) \in \mathbb{R}_{+}^{n+1}: t \geq \varepsilon>0\right\}$. We may assume that $\left\|u^{*}\right\|_{L^{p(\cdot)}\left(\mathbb{R}^{n}\right)}>0$. Indeed, observe that, for any $(x, t) \in \mathbb{R}_{+}^{n+1}$ and $y \in B(x, t),|u(x, t)| \leq u^{*}(y)$. Then, by Remark 1.2(ii), we find that, for all $(x, t) \in \mathbb{R}_{+}^{n+1}$ satisfying $|u(x, t)| \geq\left\|u^{*}\right\|_{L^{p(\cdot)}\left(\mathbb{R}^{n}\right)}$,

$$
\begin{aligned}
|u(x, t)|^{p_{-}} & =\frac{1}{|B(x, t)|} \int_{B(x, t)}\left[\frac{|u(x, t)|}{\left\|u^{*}\right\|_{L^{p(\cdot)}\left(\mathbb{R}^{n}\right)}}\right]^{p_{-}}\left\|u^{*}\right\|_{L^{p(\cdot)}\left(\mathbb{R}^{n}\right)}^{p_{-}} d y \\
& \lesssim t^{-n}\left\|u^{*}\right\|_{L^{p(\cdot)}\left(\mathbb{R}^{n}\right)}^{p_{-}} \int_{B(x, t)}\left[\frac{u^{*}(y)}{\left\|u^{*}\right\|_{L^{p(\cdot)}\left(\mathbb{R}^{n}\right)}}\right]^{p(y)} d y \lesssim t^{-n}\left\|u^{*}\right\|_{L^{p(\cdot)}\left(\mathbb{R}^{n}\right)}^{p_{-}},
\end{aligned}
$$

which shows that, for all $(x, t) \in \mathbb{R}_{+}^{n+1}$ with $t \geq \varepsilon>0$,

$$
|u(x, t)| \lesssim\left(1+\varepsilon^{-n / p_{-}}\right)\left\|u^{*}\right\|_{L^{p(\cdot)\left(\mathbb{R}^{n}\right)}} .
$$

Thus, the above claim holds true.

Now, we let $\varepsilon \in(0, \infty)$ be fixed and $f_{\varepsilon}(\cdot):=u(\cdot, \varepsilon)$. We prove that, for all $(x, t) \in \mathbb{R}_{+}^{n+1}$,

$$
f_{\varepsilon} * P_{t}(x)=u(x, t+\varepsilon)=: u_{\varepsilon}(x, t) .
$$


Indeed, from the above claim, $\left[25, \mathrm{p} .48\right.$, Theorem 2.1(b)] and the fact $f_{\varepsilon}$ is hounded and continuous, we deduce that $f_{\varepsilon} * P_{t}$ is bounded, harmonic on $\mathbb{R}_{+}^{n+1}$, continuous on the closure $\overline{\mathbb{R}_{+}^{n+1}}:=\mathbb{R}^{n} \times[0, \infty)$ and equals to $f_{\varepsilon}$ on the boundary $\mathbb{R}^{n} \times\{0\}$. On the other hand, by the assumption on $u$ and the above claim again, we know that $u(\cdot, t+\varepsilon)$ is also bounded, harmonic on $\mathbb{R}_{+}^{n+1}$, continuous on the closure $\overline{\mathbb{R}_{+}^{n+1}}:=\mathbb{R}^{n} \times[0, \infty)$ and has the same value with $f_{\varepsilon} * P_{t}$ on the boundary $\mathbb{R}^{n} \times\{0\}$. Thus, by the maximum modulus principle of harmonic functions (see [25, p. 40, Theorem 1.5]), we conclude that (2.4) holds true.

Since $u_{\varepsilon}^{*} \leq u^{*} \in L^{p(\cdot)}\left(\mathbb{R}^{n}\right)$, it follows, from Lemma 2.2, that

$$
\left\|f_{\varepsilon}\right\|_{H^{p(\cdot)\left(\mathbb{R}^{n}\right)}} \sim\left\|u_{\varepsilon}^{*}\right\|_{L^{p(\cdot)}\left(\mathbb{R}^{n}\right)} \lesssim\left\|u^{*}\right\|_{L^{p(\cdot)\left(\mathbb{R}^{n}\right)}} .
$$

This implies that $\left\{f_{\varepsilon}\right\}_{\varepsilon \in(0, \infty)}$ is uniformly bounded in $H^{p(\cdot)}\left(\mathbb{R}^{n}\right)$ and hence in $\mathcal{S}^{\prime}\left(\mathbb{R}^{n}\right)$ due to [14, Remark 3.5]. Therefore, by the weak compactness of $\mathcal{S}^{\prime}\left(\mathbb{R}^{n}\right)$ (see, for example, [25, p. 119]), we conclude that there exist a subsequence $\left\{\varepsilon_{k}\right\}_{k \in \mathbb{N}} \subset(0, \infty), \varepsilon_{k} \rightarrow 0$ as $k \rightarrow \infty$ and an element $f \in \mathcal{S}^{\prime}\left(\mathbb{R}^{n}\right)$ such that $f_{\varepsilon_{k}} \rightarrow f$ as $k \rightarrow \infty$ in $\mathcal{S}^{\prime}\left(\mathbb{R}^{n}\right)$. Moreover, for any $\Phi \in \mathcal{S}\left(\mathbb{R}^{n}\right)$ with $\int_{\mathbb{R}^{n}} \Phi(x) d x \neq 0$ and $x \in \mathbb{R}^{n}$, we have

$$
M_{\Phi,+}^{*}(f)(x):=\sup _{t \in(0, \infty)}\left|f * \Phi_{t}(x)\right|=\sup _{t \in(0, \infty)} \lim _{k \rightarrow \infty}\left|f_{\varepsilon_{k}} * \Phi_{t}(x)\right| \leq \lim _{k \rightarrow \infty} M_{\Phi,+}^{*}\left(f_{\varepsilon_{k}}\right)(x),
$$

which, combined with Lemma 2.2, the Fatou lemma (see [4, Theorem 2.61]) and (2.5), implies that

$$
\begin{aligned}
\|f\|_{H^{p(\cdot)\left(\mathbb{R}^{n}\right)}} & \sim\left\|M_{\Phi,+}^{*}(f)\right\|_{L^{p(\cdot)}\left(\mathbb{R}^{n}\right)} \lesssim\left\|\lim _{k \rightarrow \infty} M_{\Phi,+}^{*}\left(f_{\varepsilon_{k}}\right)\right\|_{L^{p(\cdot)}\left(\mathbb{R}^{n}\right)} \\
& \lesssim \liminf _{k \rightarrow \infty}\left\|M_{\Phi,+}^{*}\left(f_{\varepsilon_{k}}\right)\right\|_{L^{p(\cdot)}\left(\mathbb{R}^{n}\right)} \sim \liminf _{k \rightarrow \infty}\left\|f_{\varepsilon_{k}}\right\|_{H^{p(\cdot)}\left(\mathbb{R}^{n}\right)} \lesssim\left\|u^{*}\right\|_{L^{p(\cdot)}\left(\mathbb{R}^{n}\right)} .
\end{aligned}
$$

This shows that $f \in H^{p(\cdot)}\left(\mathbb{R}^{n}\right)$ and hence $f * P_{t}$ is well defined due to Remark 2.1. By this, (2.2), the facts that $u$ is harmonic and $\left\{f_{\varepsilon_{k}}\right\}_{k \in \mathbb{N}}$ converges to $f$ in $\mathcal{S}^{\prime}\left(\mathbb{R}^{n}\right)$, we find that, for all $(x, t) \in \mathbb{R}_{+}^{n+1}$,

$$
u(x, t)=\lim _{k \rightarrow \infty} u\left(x, t+\varepsilon_{k}\right)=\lim _{k \rightarrow \infty}\left(f_{\varepsilon_{k}} * P_{t}\right)(x)=\left(f * P_{t}\right)(x),
$$

which completes the proof of Proposition 2.5.

Let $\boldsymbol{F}:=\left\{u_{0}, u_{1}, \ldots, u_{n}\right\}$ be a vector of harmonic functions on $\mathbb{R}_{+}^{n+1}$. Then $\boldsymbol{F}$ is said to satisfy the generalized Cauchy-Riemann equation if, for all $j, k \in\{0,1, \ldots, n\}$,

$$
\left\{\begin{array}{l}
\sum_{j=0}^{n} \frac{\partial u_{j}}{\partial x_{j}}=0 \\
\frac{\partial u_{j}}{\partial x_{k}}=\frac{\partial u_{k}}{\partial x_{j}}
\end{array}\right.
$$

where, for any $(x, t) \in \mathbb{R}_{+}^{n+1}$, we let $x:=\left(x_{1}, \ldots, x_{n}\right)$ and $x_{0}:=t$.

For the harmonic vector satisfying (2.6), we have the following conclusion. 
Proposition 2.6. Let $p(\cdot) \in \mathcal{M P}\left(\mathbb{R}^{n}\right)$ with $p_{-} \in\left(\frac{n-1}{n}, \infty\right)$ and $u$ be a harmonic function on $\mathbb{R}_{+}^{n+1}$. Then $u^{*} \in L^{p(\cdot)}\left(\mathbb{R}^{n}\right)$ if and only if there exists a harmonic vector $\boldsymbol{F}:=$ $\left\{u_{0}, u_{1}, \ldots, u_{n}\right\}$ satisfying $(2.6), u_{0}=u$ and

$$
\sup _{t \in(0, \infty)}\||\boldsymbol{F}(\cdot, t)|\|_{L^{p(\cdot)}\left(\mathbb{R}^{n}\right)}<\infty
$$

here and hereafter, $|\boldsymbol{F}|:=\left(\sum_{i=0}^{n}\left|u_{i}\right|^{2}\right)^{1 / 2}$. Moreover, it holds true that

$$
\left\|u^{*}\right\|_{L^{p(\cdot)\left(\mathbb{R}^{n}\right)}} \sim \sup _{t \in(0, \infty)}\||\boldsymbol{F}(\cdot, t)|\|_{L^{p(\cdot)}\left(\mathbb{R}^{n}\right)}
$$

with the implicit equivalent positive constants independent of $u$ and $\boldsymbol{F}$.

To prove Proposition 2.6, we first establish a technical lemma with respect to the least harmonic majorant of the harmonic vector considered in Proposition 2.6 (see [25, p. 80] for the definition of the least harmonic majorant).

Lemma 2.7. Let $p(\cdot) \in \mathcal{M P}\left(\mathbb{R}^{n}\right)$ satisfy $p_{-} \in\left(\frac{n-1}{n}, \infty\right)$. If $\boldsymbol{F}$ satisfies (2.6) and (2.7), then, for any $\eta \in\left[\frac{n-1}{n}, p_{-}\right), a \in(0, \infty)$ and $(x, t) \in \mathbb{R}_{+}^{n+1}$,

$$
|\boldsymbol{F}(x, t+a)| \leq\left\{\left(|\boldsymbol{F}(\cdot, a)|^{\eta} * P_{t}\right)(x)\right\}^{1 / \eta},
$$

where $P_{t}$ denotes the Poisson kernel as in (2.1). Moreover, there exists a measurable function $g$ such that $g(x)=\lim _{a \rightarrow 0^{+}}|\boldsymbol{F}(x, a)|$ pointwise almost every $x \in \mathbb{R}^{n}$ and

$$
|\boldsymbol{F}(x, t)| \leq\left\{\left(g^{\eta} * P_{t}\right)(x)\right\}^{1 / \eta},
$$

here and hereafter, $\lim _{a \rightarrow 0^{+}}$means $a \in(0, \infty)$ and $a \rightarrow 0$.

Proof. To prove this lemma, for all $x \in \mathbb{R}^{n}, a \in[0, \infty)$ and $t \in(0, \infty)$, let $\boldsymbol{F}_{a}(x, t):=$ $\boldsymbol{F}(x, t+a)$ and, for any $q \in\left(1, \frac{p_{-}}{\eta}\right)$, let

$$
K\left(\left|\boldsymbol{F}_{a}(\cdot, t)\right|^{\eta q}, t\right):=\int_{\mathbb{R}^{n}} \frac{\left|\boldsymbol{F}_{a}(x, t)\right|^{\eta q}}{(|x|+1+t)^{n+1}} d x .
$$

We claim that $K\left(\left|\boldsymbol{F}_{a}\right|^{\eta q}, \cdot\right)$ is bounded on $(0, \infty)$. To see this, for any $t \in(0, \infty)$, write

$$
K\left(\left|\boldsymbol{F}_{a}(\cdot, t)\right|^{\eta q}, t\right)=\int_{E_{t}} \frac{|\boldsymbol{F}(x, t+a)|^{\eta q}}{(|x|+1+t)^{n+1}} d x+\int_{\left(E_{t}\right)^{\complement}} \cdots=: \text { I }+ \text { II, }
$$

where $E_{t}:=\left\{x \in \mathbb{R}^{n}:|\boldsymbol{F}(x, t+a)| \geq 1\right\}$.

Obviously, we have

$$
\mathrm{II} \leq \int_{\left(E_{t}\right)^{\complement}} \frac{1}{(|x|+1+t)^{n+1}} d x \lesssim \frac{1}{1+t} .
$$

For I, by Remark 1.2(ii) and the fact that $\eta q<p_{-}$, we find that

$$
\mathrm{I} \leq \frac{1}{(1+t)^{n+1}} \int_{E_{t}}|\boldsymbol{F}(x, t+a)|^{p(x)} d x
$$




$$
\begin{aligned}
& \leq \frac{1}{(1+t)^{n+1}} \int_{\mathbb{R}^{n}}\left[\frac{|\boldsymbol{F}(x, t+a)|}{\||\boldsymbol{F}(\cdot, t+a)|\|_{L^{p(\cdot)}\left(\mathbb{R}^{n}\right)}}\right]^{p(x)}\||\boldsymbol{F}(\cdot, t+a)|\|_{L^{p(\cdot)}\left(\mathbb{R}^{n}\right)}^{p(x)} d x \\
& \leq \frac{1}{(1+t)^{n+1}} \max \left\{\||\boldsymbol{F}(\cdot, t+a)|\|_{L^{p(\cdot)}\left(\mathbb{R}^{n}\right)}^{p_{-}},\|\boldsymbol{F}(\cdot, t+a) \mid\|_{L^{p(\cdot)}\left(\mathbb{R}^{n}\right)}^{p_{+}}\right\} \\
& \leq \frac{1}{(1+t)^{n+1}} \max \left\{\sup _{t \in(0, \infty)}\|\boldsymbol{F}(\cdot, t)\|_{L^{p(\cdot)}\left(\mathbb{R}^{n}\right)}^{p_{-}}, \sup _{t \in(0, \infty)}\|\boldsymbol{F}(\cdot, t)\|_{L^{p(\cdot)}\left(\mathbb{R}^{n}\right)}^{p_{+}}\right\} .
\end{aligned}
$$

This, together with (2.10) and (2.11), implies that, for all $t \in(0, \infty)$,

$$
K\left(\left|\boldsymbol{F}_{a}(\cdot, t)\right|^{\eta q}, t\right) \lesssim 1
$$

with the implicit positive constant independent of $t$. Therefore, the above claim holds true.

Since $\boldsymbol{F}$ satisfy (2.6), it follows, from [25, p. 234, Theorem 4.14], that, for any $\eta \in$ $\left[\frac{n-1}{n}, p_{-}\right),\left|\boldsymbol{F}_{a}\right|^{\eta}$ is subharmonic (see $[25$, p. 76] for the definition of subharmonic), with $a \in[0, \infty)$. From this, the above claim and [16, Theorem 8], we further deduce that the least harmonic majorant of $\left|\boldsymbol{F}_{a}\right|^{\eta}$ in $\mathbb{R}_{+}^{n+1}$ exists and is given by $g^{\eta} * P_{t}$, where $g$ is a measurable function and $g(x)=\lim _{t \rightarrow 0^{+}}\left|\boldsymbol{F}_{a}(x, t)\right|$ pointwise almost every $x \in \mathbb{R}^{n}$. Thus, by taking $a=0$, we then obtain (2.9); moreover, if $a \in(0, \infty)$, this, combined with $g=\left|\boldsymbol{F}_{a}(\cdot, 0)\right|=|\boldsymbol{F}(\cdot, a)|$, implies that (2.8) holds true. This finishes the proof of Lemma 2.7 .

Remark 2.8. (i) We point out that, in the proof of Lemma 2.7, the condition $p_{-} \in$ $\left(\frac{n-1}{n}, \infty\right)$ is required merely because that we need the fact that $|\boldsymbol{F}|^{\eta}$ is subharmonic on $\mathbb{R}_{+}^{n+1}$ for any $\eta \in\left[\frac{n-1}{n}, p_{-}\right)$. Thus, if there exists some $\eta_{0} \in\left(0, \frac{n-1}{n}\right)$ such that $|\boldsymbol{F}|^{\eta_{0}}$ is subharmonic on $\mathbb{R}_{+}^{n+1}$, then, for all $p_{-} \in\left(\eta_{0}, \infty\right)$, the conclusion of Lemma 2.7 still holds true. Moreover, if, for all $\eta \in(0, \infty),|\boldsymbol{F}|^{\eta}$ is subharmonic on $\mathbb{R}_{+}^{n+1}$, then, for every $p(\cdot) \in \mathcal{M P}\left(\mathbb{R}^{n}\right)$, by taking $\eta$ sufficiently small, we always have $p_{-}>\eta$ and, therefore, Lemma 2.7 holds true for every $p(\cdot) \in \mathcal{M P}\left(\mathbb{R}^{n}\right)$.

(ii) In Lemma 2.7, if $\boldsymbol{F}$ satisfies (2.6) and

$$
\sup _{t \in(0, \infty)} \int_{\mathbb{R}^{n}} \frac{|\boldsymbol{F}(x, a+t)|^{\eta q}}{(|x|+1+t)^{n+1}} d x<\infty, \quad q \in\left(1, p_{-} / \eta\right)
$$

for each $a \in[0, \infty)$, instead of $(2.7)$, then we have the same conclusion. For example, the condition that

$$
\sup _{t \in(0, \infty)}\||\boldsymbol{F}(\cdot, t)|\|_{L^{r}\left(\mathbb{R}^{n}\right)}<\infty \quad\left(r \in\left[p_{-}, \infty\right)\right)
$$

implies (2.13).

(iii) When $p(\cdot) \equiv p \in\left(\frac{n-1}{n}, \infty\right)$ is a constant, Lemma 2.7 was proved in [22, p. 122] (see also [2, Lemma 2.12]).

To prove Proposition 2.6, we also need a density result as follows. 
Lemma 2.9. Let $p(\cdot) \in \mathcal{M P}\left(\mathbb{R}^{n}\right)$. Then

(i) for all $f \in H^{p(\cdot)}\left(\mathbb{R}^{n}\right), f$ is restricted at infinity, namely, for any $r \in\left[p_{+}, \infty\right)$, it holds true that $f * \Phi \in L^{r}\left(\mathbb{R}^{n}\right)$ for all $\Phi \in \mathcal{S}\left(\mathbb{R}^{n}\right)$;

(ii) for all $r \in\left[p_{+}, \infty\right), H^{p(\cdot)}\left(\mathbb{R}^{n}\right) \cap L^{1+r}\left(\mathbb{R}^{n}\right)$ is dense in $H^{p(\cdot)}\left(\mathbb{R}^{n}\right)$. Particularly, if $p_{+} \in(0,1]$, then $H^{p(\cdot)}\left(\mathbb{R}^{n}\right) \cap L^{2}\left(\mathbb{R}^{n}\right)$ is dense in $H^{p(\cdot)}\left(\mathbb{R}^{n}\right)$.

Proof. We first prove (i). We may assume that $f \neq 0$. By the definition of the nontangential maximal function in (2.3), we see that, for all $x \in \mathbb{R}^{n}$ and $y \in B(x, 1)$,

$$
|(f * \Phi)(x)| \leq M_{\Phi, \nabla}^{*}(f)(y) .
$$

If $x \in \mathbb{R}^{n}$ satisfies that $|(f * \Phi)(x)|>\left\|M_{\Phi, \nabla}^{*}(f)\right\|_{L^{p(\cdot)}\left(\mathbb{R}^{n}\right)}$, then, by integrating on (2.14) over $B(x, 1)$, Remark $1.2($ ii) and Lemma 2.4 , we find that

$$
\begin{aligned}
|f * \Phi(x)|^{p_{-}} & \leq \frac{1}{|B(x, 1)|} \int_{B(x, 1)}\left[\frac{M_{\Phi, \nabla}^{*}(f)(y)}{\left\|M_{\Phi, \nabla}^{*}(f)\right\|_{L^{p(\cdot)}\left(\mathbb{R}^{n}\right)}}\right]^{p(y)}\left\|M_{\Phi, \nabla}^{*}(f)\right\|_{L^{p(\cdot)\left(\mathbb{R}^{n}\right)}}^{p_{-}} d y \\
& \lesssim\left\|M_{\Phi, \nabla}^{*}(f)\right\|_{L^{p(\cdot)\left(\mathbb{R}^{n}\right)}}^{p_{-}} \lesssim\|f\|_{H^{p(\cdot)\left(\mathbb{R}^{n}\right)}}^{p_{-}}<\infty
\end{aligned}
$$

Thus, $f * \Phi \in L^{\infty}\left(\mathbb{R}^{n}\right)$ and

$$
\|f * \Phi\|_{L^{\infty}\left(\mathbb{R}^{n}\right)} \lesssim\|f\|_{H^{p(\cdot)\left(\mathbb{R}^{n}\right)}} .
$$

Moreover, from (2.14) and Lemma 2.4, we deduce that

$$
\|f * \Phi\|_{L^{p(\cdot)}\left(\mathbb{R}^{n}\right)} \leq\left\|M_{\Phi, \nabla}^{*}(f)\right\|_{L^{p(\cdot)\left(\mathbb{R}^{n}\right)}} \lesssim\|f\|_{H^{p(\cdot)\left(\mathbb{R}^{n}\right)}} \cdot
$$

Let

$$
g_{1}:=f * \Phi \chi_{\left\{x \in \mathbb{R}^{n}:|f * \Phi(x)| \leq 1\right\}} \quad \text { and } \quad g_{2}:=f * \Phi \chi_{\left\{x \in \mathbb{R}^{n}:|f * \Phi(x)|>1\right\}} .
$$

Then $f * \Phi=g_{1}+g_{2}$ and, from (2.16), we easily deduce that $g_{1} \in L^{p_{+}}\left(\mathbb{R}^{n}\right)$ and $g_{2} \in$ $L^{p_{-}}\left(\mathbb{R}^{n}\right)$. By this and (2.15), we further conclude that, for any $r \in\left[p_{+}, \infty\right), f * \Phi \in L^{r}\left(\mathbb{R}^{n}\right)$ and hence $f$ is a distribution restricted at infinity.

Next, we show (ii). Let $f \in H^{p(\cdot)}\left(\mathbb{R}^{n}\right)$. Then $f$ is a bounded distribution due to Lemma 2.2 and hence, for any $t \in(0, \infty), f * P_{t}$ is well defined by Remark 2.3. By the proof of $[5$, Proposition 4.2], we know that $\left\{f * P_{t}\right\}_{t \in(0, \infty)}$ converges to $f$ in $H^{p(\cdot)}\left(\mathbb{R}^{n}\right)$. Thus, to prove (ii), we only need to show that, for any $t \in(0, \infty)$ and $r \in\left[p_{+}, \infty\right), f * P_{t} \in L^{1+r}\left(\mathbb{R}^{n}\right)$. To this end, let $\psi^{(1)}, \psi^{(2)} \in \mathcal{S}\left(\mathbb{R}^{n}\right)$ and $h \in L^{1}\left(\mathbb{R}^{n}\right)$ be as in Remark 2.1 such that (2.2) holds true. Then, for all $t \in(0, \infty), f * P_{t}=f *\left(\psi^{(1)}\right)_{t} * h_{t}+f *\left(\psi^{(2)}\right)_{t}$. By (i), we see that, for any $t \in(0, \infty)$ and $r \in\left[p_{+}, \infty\right), f *\left(\psi^{(l)}\right)_{t} \in L^{1+r}\left(\mathbb{R}^{n}\right)$ for $l \in\{1,2\}$, which, together with the Minkowski inequality for both norms and integrals, implies that

$$
\begin{aligned}
\left\|f * P_{t}\right\|_{L^{1+r}\left(\mathbb{R}^{n}\right)} & \leq\left\|f *\left(\psi^{(1)}\right)_{t} * h_{t}\right\|_{L^{1+r}\left(\mathbb{R}^{n}\right)}+\left\|f *\left(\psi^{(2)}\right)_{t}\right\|_{L^{1+r}\left(\mathbb{R}^{n}\right)} \\
& \leq\left\|f *\left(\psi^{(1)}\right)_{t}\right\|_{L^{1+r}\left(\mathbb{R}^{n}\right)}\left\|h_{t}\right\|_{L^{1}\left(\mathbb{R}^{n}\right)}+\left\|f *\left(\psi^{(2)}\right)_{t}\right\|_{L^{1+r}\left(\mathbb{R}^{n}\right)}<\infty .
\end{aligned}
$$

Therefore, for any $t \in(0, \infty), f * P_{t} \in L^{1+r}\left(\mathbb{R}^{n}\right)$, which completes the proof of (ii) and hence Lemma 2.9. 
Remark 2.10. (i) In [5, Proposition 4.2], Cruz-Uribe and Wang proved that $H^{p(\cdot)}\left(\mathbb{R}^{n}\right) \cap$ $L_{\text {loc }}^{1}\left(\mathbb{R}^{n}\right)$ is dense in $H^{p(\cdot)}\left(\mathbb{R}^{n}\right)$.

(ii) We point out that, under the assumption that $p(\cdot)$ satisfies the globally Hölder continuous condition as in Remark 1.4, by using the Calderón-Zygmund decomposition, Nakai and Sawano [14, p. 3693] also showed that $H^{p(\cdot)}\left(\mathbb{R}^{n}\right) \cap L^{1+p_{+}}\left(\mathbb{R}^{n}\right)$ is dense in $H^{p(\cdot)}\left(\mathbb{R}^{n}\right)$.

In [5, Theorem 8.4], Cruz-Uribe and Wang studied the boundedness of the convolution type Calderón-Zygmund singular integrals with sufficient regularity on $H^{p(\cdot)}\left(\mathbb{R}^{n}\right)$. As a special case of [5, Theorem 8.4], we immediately obtain the following conclusion, the details being omitted.

Lemma 2.11. Let $p(\cdot) \in \mathcal{M P}\left(\mathbb{R}^{n}\right)$. Then, for any $j \in\{1, \ldots, n\}$, the Riesz transform $R_{j}$ is bounded on $H^{p(\cdot)}\left(\mathbb{R}^{n}\right)$.

Now we are ready to prove Proposition 2.6.

Proof of Proposition 2.6. We first prove the sufficiency. Let $u$ be a harmonic function on $\mathbb{R}_{+}^{n+1}$. Assume that $\boldsymbol{F}:=\left\{u_{0}, u_{1}, \ldots, u_{n}\right\}$ satisfies $(2.6),(2.7)$ and $u_{0}:=u$. Since, for any $\eta \in\left[\frac{n-1}{n}, p_{0}\right)$ and all $t \in(0, \infty)$,

$$
\left\||\boldsymbol{F}(\cdot, t)|^{\eta}\right\|_{L^{\frac{p(\cdot)}{\eta}}\left(\mathbb{R}^{n}\right)}^{1 / \eta}=\||\boldsymbol{F}(\cdot, t)|\|_{L^{p(\cdot)}\left(\mathbb{R}^{n}\right)} \leq \sup _{t \in(0, \infty)}\||\boldsymbol{F}(\cdot, t)|\|_{L^{p(\cdot)}\left(\mathbb{R}^{n}\right)}<\infty
$$

it follows that $\left\{|\boldsymbol{F}(\cdot, t)|^{\eta}\right\}_{t \in(0, \infty)}$ is a bounded set of $L^{\frac{p(\cdot)}{\eta}}\left(\mathbb{R}^{n}\right)$. From this, the reflexivity of the Banach space $L^{\frac{p(\cdot)}{\eta}}\left(\mathbb{R}^{n}\right)$ (see [4, Corollary 2.81] and also [6, Theorem 3.4.7]) and the weak compactness of reflexive Banach spaces (see [28, p. 126, Theorem 1]), we deduce that there exist a subsequence $\left\{\left|\boldsymbol{F}\left(\cdot, t_{k}\right)\right|^{\eta}\right\}_{k \in \mathbb{N}}$ and an element $h \in L^{\frac{p(\cdot)}{\eta}}\left(\mathbb{R}^{n}\right)$ such that $t_{k} \rightarrow 0^{+}$as $k \rightarrow \infty$ and $\left\{\left|\boldsymbol{F}\left(\cdot, t_{k}\right)\right|^{\eta}\right\}_{k \in \mathbb{N}}$ converges weakly to $h$ in $L^{\frac{p(\cdot)}{\eta}}\left(\mathbb{R}^{n}\right)$. Thus, for any $g \in L^{\left(\frac{p(\cdot)}{\eta}\right)^{*}}\left(\mathbb{R}^{n}\right)$, we have

$$
\lim _{k \rightarrow \infty} \int_{\mathbb{R}^{n}}\left|\boldsymbol{F}\left(y, t_{k}\right)\right|^{\eta} g(y) d y=\int_{\mathbb{R}^{n}} h(y) g(y) d y
$$

where $\left(\frac{p(\cdot)}{\eta}\right)^{*}$ denotes the dual variable exponent of $\frac{p(\cdot)}{\eta}$. From this, it is easy to deduce that $h$ is non-negative almost everywhere in $\mathbb{R}^{n}$. For any given $(x, t) \in \mathbb{R}_{+}^{n+1}$, we let $g_{0}(y):=P_{t}(x-y)$ for all $y \in \mathbb{R}^{n}$. Then $g_{0} \in L^{\left(\frac{p(\cdot)}{\eta}\right)^{*}}\left(\mathbb{R}^{n}\right)$ and, by $(2.17)$, we obtain

$$
\lim _{k \rightarrow \infty}\left|\boldsymbol{F}\left(\cdot, t_{k}\right)\right|^{\eta} * P_{t}(x)=h * P_{t}(x),
$$

which, combined with the continuity of $|\boldsymbol{F}|$ and (2.8), implies that, for any given $(x, t) \in$ $\mathbb{R}_{+}^{n+1}$

$$
|\boldsymbol{F}(x, t)|^{\eta}=\lim _{k \rightarrow \infty}\left|\boldsymbol{F}\left(x, t_{k}+t\right)\right|^{\eta} \leq \lim _{k \rightarrow \infty}\left|\boldsymbol{F}\left(x, t_{k}\right)\right|^{\eta} * P_{t}(x)=h * P_{t}(x) .
$$


By this, the fact that $h * P_{t}(x) \lesssim \mathcal{M}(h)(x)$ for all $(x, t) \in \mathbb{R}_{+}^{n+1}, p(\cdot) \in \mathcal{M P}\left(\mathbb{R}^{n}\right)$ together with Remark 1.4(iii), (iv) and (v) of Remark 1.2, we conclude that

$$
\begin{aligned}
& \left\|u^{*}\right\|_{L^{p(\cdot)\left(\mathbb{R}^{n}\right)}} \leq\left\|\left(|\boldsymbol{F}|^{\eta}\right)^{*}\right\|_{L^{\frac{p(\cdot)}{\eta}\left(\mathbb{R}^{n}\right)}}^{1 / \eta} \lesssim\|\mathcal{M}(h)\|_{L^{\frac{p(\cdot)}{\eta}}\left(\mathbb{R}^{n}\right)}^{1 / \eta} \lesssim\|h\|_{L^{\frac{p(\cdot)}{\eta}}\left(\mathbb{R}^{n}\right)}^{1 / \eta} \\
& \sim \sup _{\|g\|_{L}(p(\cdot) / \eta)^{*}\left(\mathbb{R}^{n}\right)} \leq 1\left|\int_{\mathbb{R}^{n}} h(y) g(y) d y\right|^{1 / \eta} \\
& \left.\left.\sim \sup _{\|g\|_{L(p(\cdot) / \eta)^{*}\left(\mathbb{R}^{n}\right)} \leq 1} \lim _{k \rightarrow \infty}\left|\int_{\mathbb{R}^{n}}\right| \boldsymbol{F}\left(y, t_{k}\right)\right|^{\eta} g(y) d y\right|^{1 / \eta} \\
& \lesssim \lim _{k \rightarrow \infty}\left\|\left|\boldsymbol{F}\left(\cdot, t_{k}\right)\right|^{\eta}\right\|_{L^{\frac{p(\cdot)}{\eta}\left(\mathbb{R}^{n}\right)}}^{1 / \eta} \lesssim \sup _{t \in(0, \infty)}\||\boldsymbol{F}(\cdot, t)|\|_{L^{p(\cdot)\left(\mathbb{R}^{n}\right)}}<\infty .
\end{aligned}
$$

This implies that $u^{*} \in L^{p(\cdot)}\left(\mathbb{R}^{n}\right)$ and finishes the proof of sufficiency.

Conversely, we show the necessity. Let $u$ be a harmonic function on $\mathbb{R}_{+}^{n+1}$ and $u^{*} \in$ $L^{p(\cdot)}\left(\mathbb{R}^{n}\right)$. Then, by Proposition 2.5, there exists $f \in H^{p(\cdot)}\left(\mathbb{R}^{n}\right)$ such that $u(x, t)=f * P_{t}(x)$ for all $(x, t) \in \mathbb{R}_{+}^{n+1}$ and

$$
\|f\|_{H^{p(\cdot)}\left(\mathbb{R}^{n}\right)} \lesssim\left\|u^{*}\right\|_{L^{p(\cdot)}\left(\mathbb{R}^{n}\right)} \cdot
$$

Since $H^{p(\cdot)}\left(\mathbb{R}^{n}\right) \cap L^{1+r}\left(\mathbb{R}^{n}\right)$ with $r \in\left[p_{+}, \infty\right)$ is dense in $H^{p(\cdot)}\left(\mathbb{R}^{n}\right)$ (see Lemma 2.9), it follows that there exist $\left\{f_{k}\right\}_{k \in \mathbb{N}} \subset\left(H^{p(\cdot)}\left(\mathbb{R}^{n}\right) \cap L^{1+r}\left(\mathbb{R}^{n}\right)\right)$ such that $f_{k} \rightarrow f$ in $H^{p(\cdot)}\left(\mathbb{R}^{n}\right)$ as $k \rightarrow \infty$ and

$$
\left\|f_{k}\right\|_{H^{p(\cdot)\left(\mathbb{R}^{n}\right)}} \lesssim\|f\|_{H^{p(\cdot)\left(\mathbb{R}^{n}\right)}} \cdot
$$

For each $k \in \mathbb{N}, j \in\{1, \ldots, n\}$ and $(x, t) \in \mathbb{R}_{+}^{n+1}$, let

$$
u_{k}^{(0)}(x, t):=\left(f_{k} * P_{t}\right)(x) \text { and } u_{k}^{(j)}(x, t):=\left(f_{k} * Q_{t}^{(j)}\right)(x),
$$

where $P_{t}$ is the Poisson kernel as in (2.1) and $Q_{t}^{(j)}$ the $j$-th conjugate Poisson kernel defined by setting, for all $x \in \mathbb{R}^{n}$,

$$
Q_{t}^{(j)}(x):=\frac{\Gamma([n+1] / 2)}{\pi^{(n+1) / 2}} \frac{x_{j}}{\left(t^{2}+|x|^{2}\right)^{(n+1) / 2}} .
$$

From $\left[25\right.$, p. 236, Theorem 3.17] and the fact that $\left\{f_{k}\right\}_{k \in \mathbb{N}} \subset L^{1+r}\left(\mathbb{R}^{n}\right)$, we deduce that the harmonic vector $\boldsymbol{F}_{k}:=\left\{u_{k}^{(0)}, u_{k}^{(1)}, \ldots, u_{k}^{(n)}\right\}$ satisfies the generalized Cauchy-Riemann equation (2.6). Moreover, [21, p. 65, Theorem 3 and p.78, Item 4.4] implies that, for each $j \in\{1, \ldots, n\}$ and all $(x, t) \in \mathbb{R}_{+}^{n+1},\left(f_{k} * Q_{t}^{(j)}\right)(x)=\left(R_{j}\left(f_{k}\right) * P_{t}\right)(x)$, due to the fact that $\left\{f_{k}\right\}_{k \in \mathbb{N}} \subset L^{1+r}\left(\mathbb{R}^{n}\right)$. From this, Lemmas 2.2 and 2.11, and (2.20), we deduce that, for any $k \in \mathbb{N}$ and $j \in\{1, \ldots, n\}$,

$(2.21) \sup _{t \in(0, \infty)}\left\|u_{k}^{(j)}(\cdot, t)\right\|_{L^{p(\cdot)\left(\mathbb{R}^{n}\right)}} \lesssim\left\|R_{j}\left(f_{k}\right)\right\|_{H^{p(\cdot)}\left(\mathbb{R}^{n}\right)} \lesssim\left\|f_{k}\right\|_{H^{p(\cdot)}\left(\mathbb{R}^{n}\right)} \lesssim\|f\|_{H^{p(\cdot)}\left(\mathbb{R}^{n}\right)}$ 
and, similarly, we also have

$$
\sup _{t \in(0, \infty)}\left\|u_{k}^{(0)}(\cdot, t)\right\|_{L^{p(\cdot)\left(\mathbb{R}^{n}\right)}} \lesssim\left\|f_{k}\right\|_{H^{p(\cdot)\left(\mathbb{R}^{n}\right)}} \lesssim\|f\|_{H^{p(\cdot)\left(\mathbb{R}^{n}\right)}}
$$

By this, (2.19) and (2.21), we find that

$$
\sup _{t \in(0, \infty)}\left\|\left|\boldsymbol{F}_{k}(\cdot, t)\right|\right\|_{L^{p(\cdot)}\left(\mathbb{R}^{n}\right)} \lesssim\|f\|_{H^{p(\cdot)}\left(\mathbb{R}^{n}\right)} \lesssim\left\|u^{*}\right\|_{L^{p(\cdot)}\left(\mathbb{R}^{n}\right)} \cdot
$$

Next, we claim that there exists $\left\{k_{i}\right\}_{i \in \mathbb{N}} \subset \mathbb{N}$ such that $k_{i} \rightarrow \infty$ as $i \rightarrow \infty$ and, for all $(x, t) \in \mathbb{R}_{+}^{n+1}$,

$$
\lim _{i \rightarrow \infty} f_{k_{i}} * P_{t}(x)=f * P_{t}(x), \quad \lim _{i \rightarrow \infty} R_{j}\left(f_{k_{i}}\right) * P_{t}(x)=R_{j}(f) * P_{t}(x) .
$$

Indeed, since $\left\{f_{k}\right\}_{k \in \mathbb{N}}$ converges to $f$ in $H^{p(\cdot)}\left(\mathbb{R}^{n}\right)$, it follows, from Lemma 2.2, that

$$
\lim _{k \rightarrow \infty}\left\|f_{k} * P_{t}-f * P_{t}\right\|_{L^{p(\cdot)}\left(\mathbb{R}^{n}\right)} \lesssim \lim _{k \rightarrow \infty}\left\|f_{k}-f\right\|_{H^{p(\cdot)\left(\mathbb{R}^{n}\right)}}=0
$$

and, from Lemmas 2.2 and 2.11 , that, for any $j \in\{1, \ldots, n\}$,

$$
\begin{aligned}
& \lim _{k \rightarrow \infty}\left\|R_{j}\left(f_{k}\right) * P_{t}-R_{j}(f) * P_{t}\right\|_{L^{p(\cdot)}\left(\mathbb{R}^{n}\right)} \\
& \quad \lesssim \lim _{k \rightarrow \infty}\left\|R_{j}\left(f_{k}-f\right)\right\|_{H^{p(\cdot)}\left(\mathbb{R}^{n}\right)} \lesssim \lim _{k \rightarrow \infty}\left\|f_{k}-f\right\|_{H^{p(\cdot)}\left(\mathbb{R}^{n}\right)}=0 .
\end{aligned}
$$

By this, [4, Proposition 2.67] and Remark 2.1, we further conclude that there exists $\left\{k_{i}\right\}_{i \in \mathbb{N}} \subset \mathbb{N}$ such that $k_{i} \rightarrow \infty$ as $i \rightarrow \infty$ and (2.23) holds true for all $(x, t) \in \mathbb{R}_{+}^{n+1}$. Therefore, the above claim holds true.

On the other hand, by Lemma 2.11 , we know that $\left\{R_{j}(f)\right\}_{j=1}^{n} \subset H^{p(\cdot)}\left(\mathbb{R}^{n}\right)$, which, together with Lemma 2.2, implies that $\left\{R_{j}(f)\right\}_{j=1}^{n}$ are bounded distributions. Thus, $\left\{R_{j}(f) *\right.$ $\left.P_{t}\right\}_{j=1}^{n}$ are harmonic due to Remark 2.1 and hence $\boldsymbol{F}:=\left\{f * P_{t}, R_{1}(f) * P_{t}, \ldots, R_{n}(f) * P_{t}\right\}$ satisfies the generalized Cauchy-Riemann equation (2.6) (see [22, p. 122]).

Finally, by the above claim, the Fatou lemma (see [4, Theorem 2.61]) and (2.22), we conclude that

$$
\begin{aligned}
\sup _{t \in(0, \infty)}\||\boldsymbol{F}(\cdot, t)|\|_{L^{p(\cdot)}\left(\mathbb{R}^{n}\right)} & =\sup _{t \in(0, \infty)}\left\|\lim _{i \rightarrow \infty}\left|\boldsymbol{F}_{k_{i}}(\cdot, t)\right|\right\|_{L^{p(\cdot)\left(\mathbb{R}^{n}\right)}} \\
& \leq \sup _{t \in(0, \infty)} \liminf _{i \rightarrow \infty}\left\|\left|\boldsymbol{F}_{k_{i}}(\cdot, t)\right|\right\|_{L^{p(\cdot)}\left(\mathbb{R}^{n}\right)} \lesssim\left\|u^{*}\right\|_{L^{p(\cdot)}\left(\mathbb{R}^{n}\right)} .
\end{aligned}
$$

This finishes the proof of Proposition 2.6.

The following lemma can be proved by an argument similar to that used in the proof of [15, Theorem 3.5] (see also [30, Corollary 4.20]), the details being omitted.

Lemma 2.12. Let $p(\cdot) \in \mathcal{M P}\left(\mathbb{R}^{n}\right)$ and $p_{-} \in[1, \infty)$. Then $H^{p(\cdot)}\left(\mathbb{R}^{n}\right) \subset L^{p(\cdot)}\left(\mathbb{R}^{n}\right)$ and there exists a positive constant $C$ such that, for all $f \in H^{p(\cdot)}\left(\mathbb{R}^{n}\right)$,

$$
\|f\|_{L^{p(\cdot)}\left(\mathbb{R}^{n}\right)} \leq C\|f\|_{H^{p(\cdot)}\left(\mathbb{R}^{n}\right)} .
$$


We now turn to prove Theorem 1.5.

Proof of Theorem 1.5. We first prove "(i) $\Rightarrow\left(\right.$ ii)". Let $f \in H^{p(\cdot)}\left(\mathbb{R}^{n}\right)$. Then, by Lemma 2.9, we see that $f$ is a distribution restricted at infinity. On the other hand, by the definition of the radial grand maximal function in (1.1) and Lemma 2.11, we easily find that, for all $\epsilon \in(0, \infty)$,

$$
\begin{aligned}
& \left\|f * \phi_{\epsilon}\right\|_{L^{p(\cdot)\left(\mathbb{R}^{n}\right)}}+\sum_{j=1}^{n}\left\|R_{j}(f) * \phi_{\epsilon}\right\|_{L^{p(\cdot)}\left(\mathbb{R}^{n}\right)} \\
& \lesssim\left\|f_{N,+}^{*}\right\|_{L^{p(\cdot)}\left(\mathbb{R}^{n}\right)}+\sum_{j=1}^{n}\left\|\left(R_{j}(f)\right)_{N,+}^{*}\right\|_{L^{p(\cdot)}\left(\mathbb{R}^{n}\right)} \\
& \sim\|f\|_{H^{p(\cdot)\left(\mathbb{R}^{n}\right)}}+\sum_{j=1}^{n}\left\|R_{j}(f)\right\|_{H^{p(\cdot)}\left(\mathbb{R}^{n}\right)} \lesssim\|f\|_{H^{p(\cdot)}\left(\mathbb{R}^{n}\right)} .
\end{aligned}
$$

Furthermore, if $p_{-} \in[1, \infty)$, then, using Lemmas 2.11 and 2.12, we have

$$
\|f\|_{L^{p(\cdot)}\left(\mathbb{R}^{n}\right)}+\sum_{j=1}^{n}\left\|R_{j}(f)\right\|_{L^{p(\cdot)}\left(\mathbb{R}^{n}\right)} \lesssim\|f\|_{H^{p(\cdot)}\left(\mathbb{R}^{n}\right)}+\sum_{j=1}^{n}\left\|R_{j}(f)\right\|_{H^{p(\cdot)}\left(\mathbb{R}^{n}\right)} \lesssim\|f\|_{H^{p(\cdot)}\left(\mathbb{R}^{n}\right)},
$$

which completes the proof of "(i) $\Rightarrow$ (ii)".

Next, we show "(ii) $\Rightarrow(\mathrm{i})$ ". Suppose that $f$ is a distribution restricted at infinity and (1.2) holds true. For any $\epsilon \in(0, \infty)$, let $\boldsymbol{F}_{\epsilon}:=\left\{u_{0}^{(\epsilon)}, u_{1}^{(\epsilon)}, \ldots, u_{n}^{(\epsilon)}\right\}$, where $u_{0}^{(\epsilon)}:=f_{\epsilon} * P_{t}$, $u_{j}^{(\epsilon)}:=f_{\epsilon} * Q_{t}^{(j)}$ for each $j \in\{1, \ldots, n\}$ and $f_{\epsilon}:=f * \phi_{\epsilon}$. Since $f$ is a distribution restricted at infinity, it follows that $f * \phi_{\epsilon} \in L^{r}\left(\mathbb{R}^{n}\right)$ with $r \in\left[1+p_{+}, \infty\right)$ and hence $\left\{u_{j}^{(\epsilon)}\right\}_{j=0}^{n}$ are harmonic on $\mathbb{R}_{+}^{n+1}$, continuous and bounded on its closure (see [22, pp. 123124]). Moreover, by [25, p. 236, Theorem 4.17], we know that $\boldsymbol{F}_{\epsilon}$ satisfies the generalized Cauchy-Riemann equation (2.6) and $\sup _{t \in(0, \infty)}\left\|\left|\boldsymbol{F}_{\epsilon}(\cdot, t)\right|\right\|_{L^{r}\left(\mathbb{R}^{n}\right)}<\infty$. Thus, by Lemma 2.7 and Remark 2.8(ii), we see that, for all $\eta \in\left[\frac{n-1}{n}, p_{0}\right)$ and $(x, t) \in \mathbb{R}_{+}^{n+1}$,

$$
\left|\boldsymbol{F}_{\epsilon}(x, t)\right|^{\eta} \leq\left(\left|\boldsymbol{F}_{\epsilon}(x, 0)\right|^{\eta} * P_{t}\right)(x),
$$

where $\boldsymbol{F}_{\epsilon}(\cdot, 0):=\left\{f * \phi_{\epsilon}, R_{1}(f) * \phi_{\epsilon}, \ldots, R_{n}(f) * \phi_{\epsilon}\right\}$. By this and Remark 1.4(iii), we conclude that, for all $\varepsilon, t \in(0, \infty)$,

$$
\begin{aligned}
& \left\|\boldsymbol{F}_{\epsilon}(\cdot, t)\right\|_{L^{p(\cdot)\left(\mathbb{R}^{n}\right)}} \leq\left\|\left|\boldsymbol{F}_{\epsilon}(\cdot, 0)\right|^{\eta} * P_{t}\right\|_{L^{\frac{p(\cdot)}{\eta}\left(\mathbb{R}^{n}\right)}}^{1 / \eta} \lesssim\left\|\mathcal{M}\left(\left|\boldsymbol{F}_{\epsilon}(\cdot, 0)\right|^{\eta}\right)\right\|_{L^{\frac{p(\cdot)}{\eta}}\left(\mathbb{R}^{n}\right)}^{1 / \eta} \\
& \lesssim\left\|\left|\boldsymbol{F}_{\epsilon}(\cdot, 0)\right|^{\eta}\right\|_{L^{\frac{p(\cdot)}{\eta}}\left(\mathbb{R}^{n}\right)}^{1 / \eta} \sim\left\|\left|\boldsymbol{F}_{\epsilon}(\cdot, 0)\right|\right\|_{L^{p(\cdot)}\left(\mathbb{R}^{n}\right)} \\
& \lesssim\left\|f * \phi_{\epsilon}\right\|_{L^{p(\cdot)}\left(\mathbb{R}^{n}\right)}+\sum_{j=1}^{n}\left\|R_{j}(f) * \phi_{\epsilon}\right\|_{L^{p(\cdot)}\left(\mathbb{R}^{n}\right)} \lesssim A_{3},
\end{aligned}
$$

which, implies that $\sup _{t \in(0, \infty)}\left\|\boldsymbol{F}_{\epsilon}(\cdot, t)\right\|_{L^{p(\cdot)}\left(\mathbb{R}^{n}\right)} \lesssim A_{3}<\infty$. Thus, by Proposition 2.6, we know that $\left(f_{\epsilon}\right)_{P}^{*} \in L^{p(\cdot)}\left(\mathbb{R}^{n}\right)$ and $\left\|\left(f_{\epsilon}\right)_{P}^{*}\right\|_{L^{p(\cdot)}\left(\mathbb{R}^{n}\right)} \lesssim A_{3}$. Observe that $f * P_{t} \in L^{1+r}\left(\mathbb{R}^{n}\right)$ 
when $r \in\left[p_{+}, \infty\right)$ (see the proof of Lemma 2.9(ii)), it follows, from [25, p. 10, Theorem 1.18], that $f * P_{t} * \phi_{\epsilon}$ converges in measure to $f * P_{t}$ as $\epsilon \rightarrow \infty$, which, together with, the Riesz lemma (see, for example, [9, Theorem 1.1.13]) and Remark 2.1, implies that there exist $\left\{\epsilon_{k}\right\}_{k \in \mathbb{N}} \subset(0, \infty)$ such that $\epsilon_{k} \rightarrow 0^{+}$as $k \rightarrow \infty$, for all $(x, t) \in \mathbb{R}_{+}^{n+1}, \lim _{k \rightarrow \infty} f * P_{t} * \phi_{\epsilon_{k}}=$ $f * P_{t}$. Therefore, for all $x \in \mathbb{R}^{n}$

$$
\begin{aligned}
f_{P}^{*}(x) & =\sup _{|y-x|<t}\left|f * P_{t}(y)\right|=\sup _{|y-x|<t} \lim _{k \rightarrow \infty}\left|f_{\epsilon_{k}} * P_{t}(y)\right| \\
& \leq \lim _{k \rightarrow \infty} \sup _{|y-x|<t}\left|f_{\epsilon_{k}} * P_{t}(y)\right|=\lim _{k \rightarrow \infty}\left(f_{\epsilon_{k}}\right)_{P}^{*}(x),
\end{aligned}
$$

which, combined with Lemma 2.2 and the Fatou lemma (see [4, Theorem 2.61]), implies that $f \in H^{p(\cdot)}\left(\mathbb{R}^{n}\right)$ and

$$
\|f\|_{H^{p(\cdot)\left(\mathbb{R}^{n}\right)}} \sim\left\|f_{P}^{*}\right\|_{L^{p(\cdot)}\left(\mathbb{R}^{n}\right)} \lesssim\left\|\lim _{k \rightarrow \infty}\left(f_{\epsilon_{k}}\right)_{P}^{*}\right\|_{L^{p(\cdot)\left(\mathbb{R}^{n}\right)}} \lesssim A_{3} .
$$

This finishes the proof of "(ii) $\Rightarrow$ (i)".

Furthermore, if $p_{-} \in[1, \infty)$, then (1.3) implies that

$$
\left\{f, R_{1}(f), \ldots, R_{n}(f)\right\} \subset L^{p(\cdot)}\left(\mathbb{R}^{n}\right) \subset L^{p_{-}}\left(\mathbb{R}^{n}\right)+L^{p_{+}}\left(\mathbb{R}^{n}\right) .
$$

Let $f=f_{0,1}+f_{0,2}$ and $R_{j}(f)=f_{j, 1}+f_{j, 2}$ for all $j \in\{1, \ldots, n\}$, with $f_{j, 1} \in L^{p_{-}}\left(\mathbb{R}^{n}\right)$ and $f_{j, 2} \in L^{p_{+}}\left(\mathbb{R}^{n}\right)$ for all $j \in\{0,1, \ldots, n\}$. Then, for all $(x, t) \in \mathbb{R}_{+}^{n+1}$,

$$
\begin{aligned}
\boldsymbol{F}(x, t) & :=\left\{f * P_{t}(x), R_{1}(f) * P_{t}(x), \ldots, R_{n}(f) * P_{t}(x)\right\} \\
& =\left\{f_{0,1} * P_{t}(x)+f_{0,2} * P_{t}(x), \ldots, f_{n, 1} * P_{t}(x)+f_{n, 2} * P_{t}(x)\right\}
\end{aligned}
$$

and hence

$$
|\boldsymbol{F}(x, t)| \lesssim \sum_{j=0}^{n} \sum_{i=1}^{2}\left|f_{j, i} * P_{t}(x)\right|, \quad \sup _{t \in(0, \infty)}\left\|f_{j, 1} * P_{t}\right\|_{L^{p_{-}\left(\mathbb{R}^{n}\right)}}+\sup _{t \in(0, \infty)}\left\|f_{j, 2} * P_{t}\right\|_{L^{p_{+}\left(\mathbb{R}^{n}\right)}}<\infty,
$$

and

$$
\lim _{t \rightarrow 0^{+}} f * P_{t}=f, \quad \lim _{t \rightarrow 0^{+}} R_{j}(f) * P_{t}=R_{j}(f), j \in\{1, \ldots, n\},
$$

pointwise almost everywhere (see [4, Theorem 5.8]). Thus, by an argument similar to that used in the proof of (2.12), we conclude that (2.13) holds true for $\eta \in\left[\frac{n-1}{n}, p_{0}\right)$ and hence, by Remark 2.8(ii), we know that, for all $(x, t) \in \mathbb{R}_{+}^{n+1}$,

$$
|\boldsymbol{F}(x, t)|^{\eta} \leq\left(|\boldsymbol{F}(\cdot, 0)|^{\eta} * P_{t}\right)(x),
$$

where $\boldsymbol{F}(\cdot, 0):=\left\{f, R_{1}(f), \ldots, R_{n}(f)\right\}$. From this, $p(\cdot) \in \mathcal{M P}\left(\mathbb{R}^{n}\right)$ and Remark 1.4(iii), we further deduce that

$$
\sup _{t \in(0, \infty)}\|\boldsymbol{F}(\cdot, t)\|_{L^{p(\cdot)}\left(\mathbb{R}^{n}\right)} \lesssim \sup _{t \in(0, \infty)}\left\|\left\{|\boldsymbol{F}(\cdot, 0)|^{\eta} * P_{t}\right\}^{1 / \eta}\right\|_{L^{p(\cdot)}\left(\mathbb{R}^{n}\right)}
$$




$$
\begin{aligned}
& \lesssim\left\|\mathcal{M}\left(|\boldsymbol{F}(\cdot, 0)|^{\eta}\right)\right\|_{L^{p(\cdot) / \eta}}^{1 / \eta} \lesssim\left\||\boldsymbol{F}(\cdot, 0)|^{\eta}\right\|_{L^{p(\cdot) / \eta}}^{1 / \eta} \sim\||\boldsymbol{F}(\cdot, 0)|\|_{L^{p(\cdot)}\left(\mathbb{R}^{n}\right)} \\
& \lesssim\|f\|_{L^{p(\cdot)\left(\mathbb{R}^{n}\right)}}+\sum_{j=1}^{n}\left\|R_{j}(f)\right\|_{L^{p(\cdot)}\left(\mathbb{R}^{n}\right)} \lesssim A_{3} .
\end{aligned}
$$

Therefore, by Lemma 2.2 and Proposition 2.6, we have

$$
\|f\|_{H^{p(\cdot)}\left(\mathbb{R}^{n}\right)} \sim\left\|f_{P}^{*}\right\|_{L^{p(\cdot)}\left(\mathbb{R}^{n}\right)} \sim \sup _{t \in(0, \infty)}\|\boldsymbol{F}(\cdot, t)\|_{L^{p(\cdot)}\left(\mathbb{R}^{n}\right)} \lesssim A_{3}
$$

This finishes the proof of Theorem 1.5.

\subsection{The case $p_{-} \in\left(0, \frac{n-1}{n}\right]$}

In this subsection, we prove Theorem 1.6 and begin with some notions. Following [22, p. 133], let $m \in \mathbb{N}$ and $\left\{e_{0}, e_{1}, \ldots, e_{n}\right\}$ be an orthonormal basis of $\mathbb{R}^{n+1}$. Then the tensor product of $m$ copies of $\mathbb{R}^{n+1}$ is defined to be the set

$$
\bigotimes^{m} \mathbb{R}^{n+1}:=\left\{\boldsymbol{G}:=\sum_{j_{1}, \ldots, j_{m}=0}^{n} G_{j_{1}, \ldots, j_{m}} e_{j_{1}} \otimes \cdots \otimes e_{j_{m}}: G_{j_{1}, \ldots, j_{m}} \in \mathbb{C}\right\}
$$

where $e_{j_{1}} \otimes \cdots \otimes e_{j_{m}}$ denotes the tensor product of $e_{j_{1}}, \ldots, e_{j_{m}}$, and each $\boldsymbol{G} \in \bigotimes^{m} \mathbb{R}^{n+1}$ is called a tensor of rank $m$.

Let $G: \mathbb{R}_{+}^{n+1} \rightarrow \stackrel{m}{\bigotimes} \mathbb{R}^{n+1}$ be a tensor-valued function of rank $m$ of the form that, for all $(x, t) \in \mathbb{R}_{+}^{n+1}$,

$$
\boldsymbol{G}(x, t)=\sum_{j_{1}, \ldots, j_{m}=0}^{n} G_{j_{1}, \ldots, j_{m}}(x, t) e_{j_{1}} \otimes \cdots \otimes e_{j_{m}}
$$

with $G_{j_{1}, \ldots, j_{m}}(x, t) \in \mathbb{C}$. Then the tensor-valued function $\boldsymbol{G}$ of rank $m$ is said to be symmetric if, for any permutation $\sigma$ on $\{1, \ldots, m\}, j_{1}, \ldots, j_{m} \in\{0,1, \ldots, n\}$ and $(x, t) \in$ $\mathbb{R}^{n+1}$,

$$
G_{j_{1}, \ldots, j_{m}}(x, t)=G_{j_{\sigma(1)}, \ldots, j_{\sigma(m)}}(x, t) .
$$

For $\boldsymbol{G}$ being symmetric, $\boldsymbol{G}$ is said to be of trace zero if, for all $j_{3}, \ldots, j_{m} \in\{0,1, \ldots, n\}$ and $(x, t) \in \mathbb{R}^{n+1}$,

$$
\sum_{j=0}^{n} G_{j, j, j_{3}, \ldots, j_{m}}(x, t) \equiv 0
$$

If $\boldsymbol{G}^{(1)}, \boldsymbol{G}^{(2)} \in \stackrel{m}{\bigotimes} \mathbb{R}^{n+1}$ and

$$
G^{(l)}:=\sum_{j_{1}, \ldots, j_{m}=0}^{n} G_{j_{1}, \ldots, j_{m}}^{(l)} e_{j_{1}} \otimes \cdots \otimes e_{j_{m}}, l \in\{1,2\},
$$


then define the product of $\boldsymbol{G}^{(1)}$ and $\boldsymbol{G}^{(2)}$ by setting

$$
\left\langle\boldsymbol{G}^{(1)}, \boldsymbol{G}^{(2)}\right\rangle:=\sum_{j_{1}, \ldots, j_{m}=0}^{n} G_{j_{1}, \ldots, j_{m}}^{(1)} G_{j_{1}, \ldots, j_{m}}^{(2)} .
$$

Let $\boldsymbol{G}$ be as in (2.24). Its gradient $\nabla \boldsymbol{G}: \mathbb{R}_{+}^{n+1} \rightarrow \bigotimes^{m+1} \mathbb{R}^{n+1}$ is a tensor-valued function of rank $m+1$ of the form that, for all $(x, t) \in \mathbb{R}_{+}^{n+1}$,

$$
\begin{aligned}
\nabla \boldsymbol{G}(x, t) & =\sum_{j=0}^{n} \frac{\partial \boldsymbol{G}}{\partial x_{j}}(x, t) \otimes e_{j} \\
& =\sum_{j=0}^{n} \sum_{j_{1}, \ldots, j_{m}=0}^{n} \frac{\partial G_{j_{1}, \ldots, j_{m}}}{\partial x_{j}}(x, t) e_{j_{1}} \otimes \cdots \otimes e_{j_{m}} \otimes e_{j},
\end{aligned}
$$

here and hereafter, we always let $x_{0}:=t$. A tensor-valued function $G$ is said to satisfy the generalized Cauchy-Riemann equation if both $\boldsymbol{G}$ and $\nabla \boldsymbol{G}$ are symmetric and of trace zero. Obviously, if $m=1$, this definition of generalized Cauchy-Riemann equations is equivalent to that as in (2.6). For more details on the generalized Cauchy-Riemann equation on tensor-valued functions, we refer the reader to [24].

The following conclusion is just [1, Theorem 1].

Lemma 2.13. Let $m \in \mathbb{N}$ and $u$ be a harmonic function on $\mathbb{R}_{+}^{n+1}$. Then, for all $\eta \in$ $\left[\frac{n-1}{n+m-1}, \infty\right),\left|\nabla^{m} u\right|^{\eta}$ is subharmonic, where, for all $(x, t) \in \mathbb{R}_{+}^{n+1}$,

$$
\nabla^{m} u(x, t):=\left\{\partial^{\alpha} u(x, t)\right\}_{\alpha \in \mathbb{Z}_{+}^{n+1},|\alpha|=m}
$$

and, for all $\alpha:=\left\{\alpha_{0}, \ldots, \alpha_{n}\right\} \in \mathbb{Z}_{+}^{n+1},|\alpha|:=\sum_{j=0}^{n} \alpha_{j}, x_{0}:=t, \partial^{\alpha}:=\left(\frac{\partial}{\partial x_{0}}\right)^{\alpha_{0}} \cdots\left(\frac{\partial}{\partial x_{n}}\right)^{\alpha_{n}}$ and

$$
\left|\nabla^{m} u\right|:=\left\{\sum_{\alpha \in \mathbb{Z}_{+}^{n+1},|\alpha|=m}\left|\partial^{\alpha} u(x, t)\right|^{2}\right\}^{\frac{1}{2}} .
$$

The following lemma was proved in [26, Theorem 14.3] (see also [25]).

Lemma 2.14. Let $m \in \mathbb{N} \cap[2, \infty), G$ be a tensor-valued function of rank $m$ satisfying that both $\boldsymbol{G}$ and $\nabla \boldsymbol{G}$ are symmetric, and $\boldsymbol{G}$ is of trace zero. Then there exists a harmonic function $u$ on $\mathbb{R}_{+}^{n+1}$ such that $\nabla^{m} u=\boldsymbol{G}$, namely, for all $j_{1}, \ldots, j_{m} \in\{0,1, \ldots, n\}$ and $(x, t) \in \mathbb{R}_{+}^{n+1}$,

$$
\frac{\partial}{\partial x_{j_{1}}} \cdots \frac{\partial}{\partial x_{j_{m}}} u(x, t)=\boldsymbol{G}_{j_{1}, \ldots, j_{m}}(x, t)
$$

From Lemmas 2.13 and 2.14, we easily deduce the following conclusion.

Corollary 2.15. Let $m \in \mathbb{N} \cap[2, \infty)$ and $\boldsymbol{G}$ be a tensor-valued function of rank $m$ satisfying that both $\boldsymbol{G}$ and $\nabla \boldsymbol{G}$ are symmetric, and $\boldsymbol{G}$ is of trace zero. Then, for all $\eta \in\left[\frac{n-1}{n+m-1}, \infty\right)$, $|\boldsymbol{G}|^{\eta}$ is subharmonic on $\mathbb{R}_{+}^{n+1}$. 
Proposition 2.16. Let $p(\cdot) \in \mathcal{M P}\left(\mathbb{R}^{n}\right), m \in \mathbb{N}$ and $p_{-} \in\left(\frac{n-1}{n+m-1}, \infty\right)$. Assume that $u$ is a harmonic function on $\mathbb{R}_{+}^{n+1}$ and $\boldsymbol{G}$ is a tensor-valued function of rank $m$ satisfying generalized Cauchy-Riemann equation such that $\left\langle\boldsymbol{G}, \otimes^{m} e_{0}\right\rangle=u$ and

$$
\sup _{t \in(0, \infty)}\|\boldsymbol{G}(\cdot, t)\|_{L^{p(\cdot)\left(\mathbb{R}^{n}\right)}}<\infty
$$

Then $u^{*} \in L^{p(\cdot)}\left(\mathbb{R}^{n}\right)$ and there exists a positive constant $C$ such that

$$
\left\|u^{*}\right\|_{L^{p(\cdot)\left(\mathbb{R}^{n}\right)}} \leq C \sup _{t \in(0, \infty)}\|\boldsymbol{G}(\cdot, t)\|_{L^{p(\cdot)}\left(\mathbb{R}^{n}\right)} .
$$

Proof. Let $\eta \in\left[\frac{n-1}{n+m-1}, p_{-}\right)$. Observe that $\boldsymbol{G}$ satisfies the generalized Cauchy-Riemann equation, it follows, from Corollary 2.15, that $|\boldsymbol{G}|^{\eta}$ is subharmonic. Thus, by Lemma 2.7 and its proof, we find that, for all $a \in(0, \infty)$ and $(x, t) \in \mathbb{R}_{+}^{n+1}$,

$$
|\boldsymbol{G}(x, t+a)|^{\eta} \leq\left(|\boldsymbol{G}(\cdot, a)|^{\eta} * P_{t}\right)(x) .
$$

On the other hand, it is easy to see that

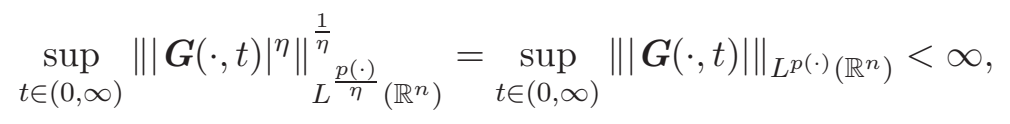

namely, $\left\{|\boldsymbol{G}(\cdot, t)|^{\eta}\right\}_{t \in(0, \infty)}$ is bounded in $L^{\frac{p(\cdot)}{\eta}}\left(\mathbb{R}^{n}\right)$. Then, by an argument similar to that used in the proof of Proposition 2.6, we conclude that there exist a subsequence $\left\{\boldsymbol{G}\left(\cdot, t_{k}\right)\right\}_{k \in \mathbb{N}}$ and $h \in L^{\frac{p(\cdot)}{\eta}}\left(\mathbb{R}^{n}\right)$ such that $h$ is non-negative almost everywhere in $\mathbb{R}^{n}$ and, for all $(x, t) \in \mathbb{R}_{+}^{n+1}$,

$$
|\boldsymbol{G}(x, t)|^{\eta}=\lim _{k \rightarrow \infty}\left|\boldsymbol{G}\left(x, t_{k}+t\right)\right|^{\eta} \leq \lim _{k \rightarrow \infty}\left|\boldsymbol{G}\left(x, t_{k}\right)\right|^{\eta} * P_{t}(x)=h * P_{t}(x) .
$$

Moreover, by this, we see that, for all $x \in \mathbb{R}^{n}$,

$$
\begin{aligned}
u^{*}(x) & =\sup _{|y-x|<t}|u(y, t)|=\sup _{|y-x|<t}\left|\left\langle\boldsymbol{G}(y, t), \otimes^{m} e_{0}\right\rangle\right| \\
& \leq \sup _{|y-x|<t}|\boldsymbol{G}(y, t)| \lesssim[\mathcal{M}(h)(x)]^{\frac{1}{\eta}}
\end{aligned}
$$

and hence, by an argument similar to that used in the proof of (2.18), we find that

$$
\left\|u^{*}\right\|_{L^{p(\cdot)\left(\mathbb{R}^{n}\right)}} \lesssim\left\|[\mathcal{M}(h)]^{\frac{1}{\eta}}\right\|_{L^{p(\cdot)\left(\mathbb{R}^{n}\right)}} \lesssim \sup _{t \in(0, \infty)}\||\boldsymbol{G}(\cdot, t)|\|_{L^{p(\cdot)}\left(\mathbb{R}^{n}\right)}
$$

This finishes the proof of Proposition 2.16.

We now prove Theorem 1.6. 
Proof of Theorem 1.6. To prove this theorem, it suffices to show "(ii) $\Rightarrow(\mathrm{i})$ ", since the proof of "(i) $\Rightarrow$ (ii)" is similar to that of Theorem 1.5.

For all $\varepsilon \in(0, \infty)$ and $(x, t) \in \mathbb{R}_{+}^{n+1}$, let $f_{\varepsilon}(x):=f * \phi_{\varepsilon}(x)$,

$$
G_{j_{1}, \ldots, j_{m}}^{(\varepsilon)}(x, t):=\left(R_{j_{1}} \cdots R_{j_{m}}\left(f_{\varepsilon}\right) * P_{t}\right)(x)
$$

and

$$
\boldsymbol{G}^{(\varepsilon)}(x, t):=\sum_{j_{1}, \ldots, j_{m}=0}^{\infty} G_{j_{1}, \ldots, j_{m}}^{(\varepsilon)}(x, t) e_{j_{1}} \otimes \cdots \otimes e_{j_{m}},
$$

where $P_{t}$ denotes the Poisson kernel as in (2.1) and $R_{0}:=I$ denotes the identity operator. Since $f * \phi_{\varepsilon} \in L^{1+r}\left(\mathbb{R}^{n}\right)$ with $r \in\left[p_{+}, \infty\right)$ due to Lemma 2.9(i), it follows, from the Fourier transform, that both $\boldsymbol{G}^{(\varepsilon)}$ and $\nabla \boldsymbol{G}^{(\varepsilon)}$ are symmetric, and $\boldsymbol{G}^{(\varepsilon)}$ is of trace zero. Then, by Proposition 2.16, we find that $\left(f_{\varepsilon}\right)_{P}^{*} \in L^{p(\cdot)}\left(\mathbb{R}^{n}\right)$ and $\left\|\left(f_{\varepsilon}\right)_{P}^{*}\right\|_{L^{p(\cdot)}\left(\mathbb{R}^{n}\right)} \lesssim A_{4}$. On the other hand, by the proof of Lemma 2.9(ii), we see that $f * P_{t} \in L^{1+r}\left(\mathbb{R}^{n}\right)$. From this, $[25$, p. 10, Theorem 1.18] and the fact that $\int_{\mathbb{R}^{n}} \phi(x) d x=1$, we deduce that $f * P_{t} * \phi_{\varepsilon}$ converges to $f * P_{t}$ in measure as $\varepsilon \rightarrow 0$, which, combined with the Riesz lemma (see, for example, [9, Theorem 1.1.13]), implies that, there exist $\left\{\varepsilon_{k}\right\}_{k \in \mathbb{N}} \subset(0, \infty)$ such that $\varepsilon_{k} \rightarrow 0^{+}$as $k \rightarrow \infty$ and, for any given $(x, t) \in \mathbb{R}_{+}^{n+1}$,

$$
\lim _{k \rightarrow \infty} f_{\varepsilon_{k}} * P_{t}(x)=\lim _{k \rightarrow \infty} f * P_{t} * \phi_{\varepsilon_{k}}(x)=f * P_{t}(x) .
$$

From this, we further deduce that, for all $x \in \mathbb{R}^{n}$,

$$
\begin{aligned}
f_{P}^{*}(x) & =\sup _{|y-x|<t}\left|f * P_{t}(y)\right|=\sup _{|y-x|<t} \lim _{k \rightarrow \infty}\left|f_{\varepsilon_{k}} * P_{t}(y)\right| \\
& \leq \lim _{k \rightarrow \infty} \sup _{|y-x|<t}\left|f_{\varepsilon_{k}} * P_{t}(y)\right|=\lim _{k \rightarrow \infty}\left(f_{\varepsilon_{k}}\right)_{P}^{*}(x) .
\end{aligned}
$$

By this and the Fatou lemma ([4, Theorem 2.61]), we conclude that

$$
\left\|f_{P}^{*}\right\|_{L^{p(\cdot)}\left(\mathbb{R}^{n}\right)} \leq\left\|\lim _{k \rightarrow \infty}\left(f_{\varepsilon_{k}} * P_{t}\right)^{*}\right\|_{L^{p(\cdot)\left(\mathbb{R}^{n}\right)}} \leq \liminf _{k \rightarrow \infty}\left\|\left(f_{\varepsilon_{k}}\right)_{P}^{*}\right\|_{L^{p(\cdot)}\left(\mathbb{R}^{n}\right)} \lesssim A_{4} .
$$

Therefore, $f \in H^{p(\cdot)}\left(\mathbb{R}^{n}\right)$ and $\|f\|_{H^{p(\cdot)}\left(\mathbb{R}^{n}\right)} \lesssim A_{4}$, which completes the proof of Theorem 1.6 .

Acknowledgements. The authors would like to express their deep thanks to Professor Yoshihiro Sawano for several useful conversations on the subject of this article.

\section{References}

[1] A.-P. Calderón and A. Zygmund, On higher gradients of harmonic functions, Studia Math. (24) 1964, 211-226.

[2] J. Cao, D.-C. Chang, D. Yang and S. Yang, Riesz transform characterizations of Musielak-Orlicz-Hardy spaces, Trans. Amer. Math. Soc. (to appear) or arXiv: 1401.7373. 
[3] R. R. Coifman and G. Weiss, Extensions of Hardy spaces and their use in analysis, Bull. Amer. Math. Soc. 83 (1977), 569-645.

[4] D. V. Cruz-Uribe and A. Fiorenza, Variable Lebesgue Spaces. Foundations and Harmonic Analysis, Applied and Numerical Harmonic Analysis, Birkhäuser/Springer, Heidelberg, 2013.

[5] D. Cruz-Uribe and L.-A. D. Wang, Variable Hardy spaces, Indiana Univ. Math. J. 63 (2014), 447-493.

[6] L. Diening, P. Harjulehto, P. Hästö and M. Růžička, Lebesgue and Sobolev Spaces with Variable Exponents, Lecture Notes in Mathematics, 2017, Springer, Heidelberg, 2011.

[7] C. Fefferman and E. M. Stein, $H^{p}$ spaces of several variables, Acta Math. 129 (1972), 137-193.

[8] D. Goldberg, A local version of real Hardy spaces, Duke Math. J. 46 (1979), 27-42.

[9] L. Grafakos, Classical Fourier Analysis, Third edition, Graduate Texts in Mathematics, 249, Springer, New York, 2014.

[10] M. Izuki, E. Nakai and Y. Sawano, Function spaces with variable exponents - an introduction-, Sci. Math. Jpn. 77 (2014), 187-315.

[11] L. D. Ky, New Hardy spaces of Musielak-Orlicz type and boundedness of sublinear operators, Integral Equations Operator Theory 78 (2014), 115-150.

[12] B. Muckenhoupt and R. L. Wheeden, On the dual of weighted $H^{1}$ of the half-space, Studia Math. 63 (1978), 57-79.

[13] S. Müller, Hardy space methods for nonlinear partial differential equations, Tatra Mt. Math. Publ. 4 (1994), 159-168.

[14] E. Nakai and Y. Sawano, Hardy spaces with variable exponents and generalized Campanato spaces, J. Funct. Anal. 262 (2012), 3665-3748.

[15] E. Nakai and Y. Sawano, Orlicz-Hardy spaces and their duals, Sci. China Math. 57 (2014), 903-962.

[16] S. Nualtaranee, On least harmonic majorants in half-spaces, Proc. London Math. Soc. (3) 27 (1973), 243-260.

[17] W. Orlicz, Über konjugierte Exponentenfolgen, Studia Math. 3 (1931), 200-211.

[18] M. M. Peloso and S. Secco, Local Riesz transforms characterization of local Hardy spaces, Collect. Math. 59 (2008), 299-320.

[19] Y. Sawano, Atomic decompositions of Hardy spaces with variable exponents and its application to bounded linear operators, Integral Equations Operator Theory 77 (2013), 123-148.

[20] E. M. Stein, On the theory of harmonic functions of several variables. II. Behavior near the boundary, Acta Math. 106 (1961), 137-174.

[21] E. M. Stein, Singular Integrals and Differentiability Properties of Functions, Princeton Mathematical Series, No. 30. Princeton University Press, Princeton, N.J. 1970.

[22] E. M. Stein, Harmonic Analysis: Real-Variable Methods, Orthogonality, and Oscillatory Integrals, Princeton Mathematical Series, No. 43. Monographs in Harmonic Analysis, III, Princeton University Press, Princeton, N.J., 1993. 
[23] E. M. Stein and G. Weiss, On the theory of harmonic functions of several variables. I. The theory of $H^{p}$-spaces, Acta Math. 103 (1960), 25-62.

[24] E. M. Stein and G. Weiss, Generalization of the Cauchy-Riemann equations and representations of the rotation group, Amer. J. Math. 90 (1968), 163-196.

[25] E. M. Stein and G. Weiss, Introduction to Fourier Analysis on Euclidean Spaces, Princeton Mathematical Series, No. 32. Princeton University Press, Princeton, N.J., 1971.

[26] A. Uchiyama, Hardy Spaces on the Euclidean Space, Springer Monographs in Mathematics. Springer-Verlag, Tokyo, 2001.

[27] R. L. Wheeden, A boundary value characterization of weighted $H^{1}$, Enseignement Math. (2) 22 (1976), 121-134.

[28] K. Yosida, Functional Analysis, Sixth edition. Grundlehren der Mathematischen Wissenschaften, Band 123. Springer-Verlag, Berlin-New York, 1978.

[29] D. Yang, W. Yuan and C. Zhuo, Musielak-Orlicz Besov-type and Triebel-Lizorkintype spaces, Rev. Mat. Complut. 27 (2014), 93-157.

[30] C. Zhuo, Y. Sawano and D. Yang, Hardy spaces with variable exponents on RDspaces and applications, Submitted.

[31] C. Zhuo, D. Yang and Y. Liang, Intrinsic square function characterizations of Hardy spaces with variable exponents, Bull. Malays. Math. Sci. Soc. (2) (to appear) or arXiv: 1411.5535 .

Dachun Yang and Ciqiang Zhuo (Corresponding author)

School of Mathematical Sciences, Beijing Normal University, Laboratory of Mathematics and Complex Systems, Ministry of Education, Beijing 100875, People's Republic of China E-mails: dcyang@bnu.edu.cn (D. Yang) cqzhuo@mail. bnu.edu.cn (C. Zhuo)

Eiichi Nakai

Department of Mathematics, Ibaraki University, Mito, Ibaraki 310-8512, Japan

E-mail: enakai@mx.ibaraki.ac.jp (E. Nakai) 